\title{
Existence and uniqueness results for the coupled systems of implicit fractional differential equations with periodic boundary conditions
}

Wei Zhang ${ }^{1}$, Wenbin Liu ${ }^{1 *}$ and Tingting Xue

${ }^{*}$ Correspondence:

wblium@163.com

'School of Mathematics, China

University of Mining and

Technology, Xuzhou, P.R. China

\begin{abstract}
In this paper, we study the periodic boundary value problems for the coupled systems of fractional implicit differential equations. Basing on the coincidence degree theory, we establish the existence and uniqueness theorems. Further, we provide several examples to show our main results.
\end{abstract}

MSC: 34A08; 34B15

Keywords: Fractional implicit differential equation; Coupled system; Periodic boundary value problem; Existence and uniqueness; Coincidence degree theory

\section{Introduction}

In the past two decades, there has been tremendous interest in studying fractional differential equations (FDEs for short) due to their extensive applications in various fields of engineering and scientific disciplines (see [1-8]). For example, in [8], Laskin proposed the following fractional stochastic dynamic model for the considered market:

$$
\left\{\begin{array}{l}
D_{0+}^{\mu} x(t)=\lambda x(t)+F(t), \quad 0<\mu \leq 1, \\
D_{0+}^{\mu-1} x(0)=x_{0}
\end{array}\right.
$$

where $D_{0+}^{\mu}$ is the standard Riemann-Liouville fractional derivative of order $\mu, \lambda$ and $F(t)$ respectively denote the expected rate and the random force.

As an important issue for the theory of FDEs, the existence, uniqueness, and multiplicity of solutions for the nonlinear fractional initial value problems (FIVPs for short) and fractional boundary value problems (FBVPs for short) have attracted scholars' attention. For some recent work on the topic, see papers [9-19], monographs [1, 2, 20, 21], and the references therein. In particular, many researchers focused on studying the FDEs with periodic boundary conditions (PBCs for short) (see [22-30]).

(c) The Author(s) 2018. This article is distributed under the terms of the Creative Commons Attribution 4.0 International License (http://creativecommons.org/licenses/by/4.0/), which permits unrestricted use, distribution, and reproduction in any medium, provided you give appropriate credit to the original author(s) and the source, provide a link to the Creative Commons license, and indicate if changes were made. 
In [22], Cabada and Kisela discussed the following FDE with PBC:

$$
\left\{\begin{array}{l}
D_{0+}^{\alpha} u(t)-\lambda u(t)=f\left(t, t^{1-\alpha} u(t)\right), \quad 0<\alpha \leq 1, \\
\lim _{t \rightarrow 0^{+}} t^{1-\alpha} u(t)=u(1),
\end{array}\right.
$$

where $\lambda \neq 0(\lambda \in \mathbb{R}), D_{0+}^{\alpha}$ is the standard Riemann-Liouville fractional derivative of order $\alpha$. The existence results were based on the fixed point theorems and monotone iterative technique.

In [23], Staněk dealt with the following FDE with PBC:

$$
\left\{\begin{array}{l}
{ }^{c} D^{\alpha} u(t)+q(t, u(t))^{c} D^{\beta} u(t)=f(t, u(t)), \quad 0<\beta<\alpha \leq 1, \\
u(0)=u(T), \quad T>0,
\end{array}\right.
$$

where ${ }^{c} D^{(\cdot)}$ is the Caputo fractional derivative of fractional order. The existence, multiplicity, and uniqueness results were proved by the Schauder fixed point theorem.

Recently, some scholars have considered very interesting aspects of IVPs and BVPs for the implicit FDEs (see [29-39]). For example, Nieto, Ouahab, and Venktesh [32] investigated a class of implicit FIVP:

$$
\left\{\begin{array}{l}
{ }^{c} D^{\alpha} y(t)=f\left(t, y(t),{ }^{c} D^{\alpha} y(t)\right), \quad t \in J, 0<\alpha<1, \\
y(0)=y_{0}
\end{array}\right.
$$

where $J=[0, b], b>0,{ }^{c} D^{\alpha}$ is the Caputo fractional derivative of order $\alpha$, and $f: J \times \mathbb{R}^{2} \rightarrow$ $\mathbb{R}$ is a continuous function. By using fixed point theory and approximation method, the existence and uniqueness results were obtained.

In [29], Benchohra, Bouriah, and Graef studied the following implicit FDE with PBC:

$$
\left\{\begin{array}{l}
{ }^{c} D^{\alpha} y(t)=f\left(t, y(t),{ }^{c} D^{\alpha} y(t)\right), \quad t \in J, 0<\alpha \leq 1, \\
y(0)=y(T), \quad T>0,
\end{array}\right.
$$

where $J=[0, T],{ }^{c} D^{\alpha}$ is the Caputo fractional derivative of order $\alpha$ and $f: J \times \mathbb{R}^{2} \rightarrow \mathbb{R}$ is a continuous function. Applying the coincidence degree theory, an existence result was given.

In [33], Ali, Zada, and Shah proved the existence and uniqueness of the solutions for the following implicit FDEs with three-point BCs:

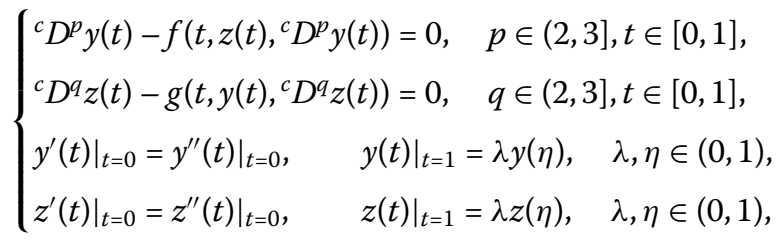

where ${ }^{c} D^{(\cdot)}$ is the Caputo fractional derivative of fractional order and $f, g: J \times \mathbb{R}^{2} \rightarrow \mathbb{R}$ are continuous functions. The results were accomplished by means of the Leray-Schauder fixed point theorem and Banach contraction principle. 
Inspired by the above work, in this paper we are mainly concerned with the existence and uniqueness of solutions for the following coupled system of nonlinear implicit FDEs with PBCs:

$$
\left\{\begin{array}{l}
D_{0+}^{\alpha} x(t)=f\left(t, t^{1-\beta} y(t), D_{0+}^{\beta} y(t)\right), \quad t \in[0,1], 0<\alpha, \beta \leq 1, \\
D_{0+}^{\beta} y(t)=g\left(t, t^{1-\alpha} x(t), D_{0+}^{\alpha} x(t)\right), \\
\lim _{t \rightarrow 0^{+}} t^{1-\alpha} x(t)=x(1), \quad \lim _{t \rightarrow 0^{+}} t^{1-\beta} y(t)=y(1),
\end{array}\right.
$$

where $D_{0+}^{(\cdot)}$ is the standard Riemann-Liouville fractional derivative of fractional order, $f, g$ : $[0,1] \times \mathbb{R}^{2} \rightarrow \mathbb{R}$ are two continuous functions. To state our main results, we assume that the nonlinear terms $f$ and $g$ satisfy the following general conditions:

$\left(\mathrm{A}_{1}\right)$ There exist nonnegative continuous functions $\gamma_{i}(t), \eta_{i}(t), \omega_{i}(t), i=1,2$, such that, for any $t \in[0,1], u_{i}, v_{i} \in \mathbb{R},(i=1,2)$,

$$
\begin{aligned}
& \left|f\left(t, t^{1-\beta} u_{1}, v_{1}\right)\right| \leq \gamma_{1}(t)\left|t^{1-\beta} u_{1}\right|+\eta_{1}(t)\left|v_{1}\right|+\omega_{1}(t), \\
& \left|g\left(t, t^{1-\alpha} u_{2}, v_{2}\right)\right| \leq \gamma_{2}(t)\left|t^{1-\alpha} u_{2}\right|+\eta_{2}(t)\left|v_{2}\right|+\omega_{2}(t) .
\end{aligned}
$$

$\left(\mathrm{A}_{2}\right)$ There exist nonnegative continuous functions $p_{i}(t), q_{i}(t), i=1,2$, such that, for any $t \in[0,1], u_{i j}, v_{i j} \in \mathbb{R},(i, j=1,2)$,

$$
\begin{aligned}
& \left|f\left(t, t^{1-\beta} u_{11}, v_{11}\right)-f\left(t, t^{1-\beta} u_{12}, v_{12}\right)\right| \leq p_{1}(t) t^{1-\beta}\left|u_{11}-u_{12}\right|+q_{1}(t)\left|v_{11}-v_{12}\right| \\
& \left|g\left(t, t^{1-\alpha} u_{21}, v_{21}\right)-g\left(t, t^{1-\alpha} u_{22}, v_{22}\right)\right| \leq p_{2}(t) t^{1-\alpha}\left|u_{21}-u_{22}\right|+q_{2}(t)\left|v_{21}-v_{22}\right| .
\end{aligned}
$$

$\left(\mathrm{A}_{3}\right)$ There exist constants $a, c>0, b, d \geq 0$ such that, for any $t \in[0,1], u_{i j}, v_{i j} \in \mathbb{R},(i, j=$ $1,2)$,

$$
\begin{aligned}
& \left|f\left(t, t^{1-\beta} u_{11}, v_{11}\right)-f\left(t, t^{1-\beta} u_{12}, v_{12}\right)\right| \geq a t^{1-\beta}\left|u_{11}-u_{12}\right|-b\left|v_{11}-v_{12}\right|, \\
& \left|g\left(t, t^{1-\alpha} u_{21}, v_{21}\right)-g\left(t, t^{1-\alpha} u_{22}, v_{22}\right)\right| \geq c t^{1-\alpha}\left|u_{21}-u_{22}\right|-d\left|v_{21}-v_{22}\right| .
\end{aligned}
$$

Remark 1.1 Condition $\left(\mathrm{A}_{2}\right)$ implies condition $\left(\mathrm{A}_{1}\right)$.

The objective of this paper is twofold. The first one is to study the existence solutions for BVP (1.1), the other is to consider the uniqueness of solution for (1.1). Our work presented in this paper has the following features. Firstly, this article generalizes the results of papers $[29,30]$ into coupled systems. Secondly, compared with $[29,30]$, we not only discuss the existence result but also establish the uniqueness result. In addition, the existence results of papers $[29,30]$ are based on condition $\left(A_{2}\right)$, in our paper the existence result can also be obtained under condition $\left(\mathrm{A}_{1}\right)$. Thirdly, we present two prior estimation ways in using Theorem 2.1 (see Sect. 2) to establish the existence results. It should be pointed out that a number of papers by applying Theorem 2.1 to solve fractional resonance boundary value problems usually used the second way (see Lemma 3.4 in Sect. 3) to estimate the prior bounds. For example [40-43]. Our results show that the first way is better than the second. We finally remark that our paper investigates the FBVP in the frame of Riemann-Liouville fractional derivative which is more complicated than such a problem involving Caputo 
fractional derivative, and if $\alpha=\beta=1$, then BVP (1.1) can be reduced to the implicit first order differential systems with PBCs.

The rest of this paper is built up as follows. We devote Sect. 2 to recalling some preliminary definitions and lemmas. We establish the existence and uniqueness theorems for problem (1.1) in Sect. 3. In order to fully explain our main results, we provide three examples in Sect. 4. Finally, we present some conclusions in Sect. 5.

\section{Preliminaries}

In this section, we recall some basic definitions, lemmas, and theorems which are used throughout this paper. Firstly, we introduce some definitions and results on fractional calculus $[1,2,44]$.

Definition 2.1 The Riemann-Liouville fractional integral of order $\alpha>0$ for a function $x: \mathbb{R}^{+} \rightarrow \mathbb{R}$ is given by

$$
I_{0+}^{\alpha} x(t)=\frac{1}{\Gamma(\alpha)} \int_{0}^{t}(t-s)^{\alpha-1} x(s) d s
$$

provided that the right-hand side integral is pointwise defined on $(0,+\infty)$.

Definition 2.2 The Riemann-Liouville fractional derivative of order $\alpha>0$ for a function $x: \mathbb{R}^{+} \rightarrow \mathbb{R}$ is given by

$$
D_{0+}^{\alpha} x(t)=\frac{d^{n}}{d t^{n}} I_{0+}^{n-\alpha} x(t)=\frac{1}{\Gamma(n-\alpha)} \frac{d^{n}}{d t^{n}} \int_{0}^{t}(t-s)^{n-\alpha-1} x(s) d s,
$$

where $n=[\alpha]+1$, provided that the right-hand side integral is pointwise defined on $(0,+\infty)$.

Lemma 2.1 Let $\alpha>0$. If $x, D_{0+}^{\alpha} x \in L^{1}(0,1)$, then

$$
I_{0+}^{\alpha} D_{0+}^{\alpha} x(t)=x(t)+c_{1} t^{\alpha-1}+c_{2} t^{\alpha-2}+\cdots+c_{n} t^{\alpha-n}
$$

where $n=[\alpha]+1, c_{i} \in \mathbb{R}(i=1,2, \ldots, n)$ are arbitrary constants.

Lemma 2.2 Let $\alpha>\beta>0$. If $x \in L^{1}(0,1)$, then

$$
I_{0+}^{\alpha} I_{0+}^{\beta} x(t)=I_{0+}^{\alpha+\beta} x(t), \quad D_{0+}^{\beta} I_{0+}^{\alpha} x(t)=I_{0+}^{\alpha-\beta} x(t),
$$

in particular $D_{0+}^{\alpha} I_{0+}^{\alpha} x(t)=x(t)$.

Lemma 2.3 (see [44]) If $\alpha>0, \lambda>-1, t>0$, then

$$
I_{0+}^{\alpha} t^{\lambda}=\frac{\Gamma(\lambda+1)}{\Gamma(\lambda+1+\alpha)} t^{\alpha+\lambda}, \quad D_{0+}^{\alpha} t^{\lambda}=\frac{\Gamma(\lambda+1)}{\Gamma(\lambda+1-\alpha)} t^{\lambda-\alpha},
$$

in particular $D_{0+}^{\alpha} t^{\alpha-m}=0, m=1,2, \ldots, n$, where $n=[\alpha]+1$. 
We recall now the basic knowledge on the coincidence degree theory. For more details, we refer the readers to [45-47].

Let $\left(X,\|\cdot\|_{X}\right)$ and $\left(Y,\|\cdot\|_{Y}\right)$ be two real Banach spaces. Suppose $L: \operatorname{dom} L \subset X \rightarrow Y$ is a Fredholm operator with index zero, then there exist two continuous projectors $P: X \rightarrow X$ and $Q: Y \rightarrow Y$ such that

$$
\operatorname{Im} P=\operatorname{Ker} L, \quad \operatorname{Im} L=\operatorname{Ker} Q, \quad X=\operatorname{Ker} L \oplus \operatorname{Ker} P, \quad Y=\operatorname{Im} L \oplus \operatorname{Im} Q,
$$

and $\left.L\right|_{\operatorname{dom}} L \cap \operatorname{Ker} P: \operatorname{dom} L \rightarrow \operatorname{Im} L$ is invertible. We denote by $K_{P}=\left(\left.L\right|_{\operatorname{dom} L \cap \operatorname{Ker} P}\right)^{-1}$. Let $\Omega$ be an open bounded subset of $X$ and $\operatorname{dom} L \cap \bar{\Omega} \neq \emptyset$. The map $N: X \rightarrow Y$ is called $L$-compact on $\bar{\Omega}$ if $Q N(\bar{\Omega})$ is bounded and $K_{P}(I-Q) N: \bar{\Omega} \rightarrow X$ is compact.

Theorem 2.1 Let $L: \operatorname{dom} L \subset X \rightarrow Y$ be a Fredholm operator of index zero and $N: X \rightarrow Y$ be L-compact on $\bar{\Omega}$. If the following conditions are satisfied:

(i) $L u \neq \lambda N u$ for any $u \in(\operatorname{dom} L \backslash \operatorname{Ker} L) \cap \partial \Omega, \lambda \in(0,1)$;

(ii) $N u \notin \operatorname{Im} L$ for any $u \in \operatorname{Ker} L \cap \partial \Omega$;

(iii) $\operatorname{deg}\left\{\left.Q N\right|_{\operatorname{Ker} L}, \Omega \cap \operatorname{Ker} L, 0\right\} \neq 0$;

then the equation $L x=N x$ has at least one solution in $\operatorname{dom} L \cap \bar{\Omega}$.

Theorem 2.2 Let $L: \operatorname{dom} L \subset X \rightarrow Y$ be a Fredholm operator of index zero, $\Omega \subset X$ be an open bounded set symmetric with $0 \in \Omega$ and $N: \bar{\Omega} \rightarrow Y$ is L-compact. If $L x-N x \neq$ $\lambda(-L x-N(-x))$ for all $(\lambda, x) \in(0,1] \times \operatorname{dom} L \cap \partial \Omega$, then $L x=N x$ has a solution in $\operatorname{dom} L \cap \bar{\Omega}$.

\section{Main results}

Take

$$
X_{1}=\left\{x: t^{1-\alpha} x, D_{0+}^{\alpha} x \in C[0,1]\right\}, \quad X_{2}=\left\{y: t^{1-\beta} y, D_{0+}^{\beta} y \in C[0,1]\right\},
$$

endowed with the norms

$$
\|x\|_{X_{1}}=\left\|t^{1-\alpha} x\right\|_{\infty}+\left\|D_{0+}^{\alpha} x\right\|_{\infty}, \quad\|y\|_{X_{2}}=\left\|t^{1-\beta} y\right\|_{\infty}+\left\|D_{0+}^{\beta} y\right\|_{\infty},
$$

respectively, where $\|\cdot\|_{\infty}=\max _{t \in[0,1]}|\cdot|$. We can easily check that $\left(X_{1},\|\cdot\|_{X_{1}}\right)$ and $\left(X_{2}\right.$, $\left.\|\cdot\|_{X_{2}}\right)$ are two Banach spaces. Let $Z_{1}=C[0,1]$ with norm $\|z\|_{Z_{1}}=\max _{t \in[0,1]}|z(t)|$. According to the basic theory of functional analysis, we have $X=X_{1} \times X_{2}$ and $Z=Z_{1} \times Z_{1}$ are also Banach spaces, respectively, with the norms

$$
\|(x, y)\|_{X}=\max \left\{\|x\|_{X_{1}},\|y\|_{X_{2}}\right\}, \quad\|(u, v)\|_{Z}=\max \left\{\|u\|_{Z_{1}},\|v\|_{Z_{1}}\right\} .
$$

Define the linear operators $L_{i}: \operatorname{dom} L_{i} \subset X_{i} \rightarrow Z_{1}(i=1,2)$ and the nonlinear operators $N_{1}: X_{2} \rightarrow Z_{1}, N_{2}: X_{1} \rightarrow Z_{1}$ by

$$
\begin{array}{llll}
L_{1} x(t)=D_{0+}^{\alpha} x(t), & x(t) \in \operatorname{dom} L_{1}, & N_{1} y(t)=f\left(t, t^{1-\beta} y(t), D_{0+}^{\beta} y(t)\right), & y(t) \in X_{2}, \\
L_{2} y(t)=D_{0+}^{\beta} y(t), & y(t) \in \operatorname{dom} L_{2}, & N_{2} x(t)=g\left(t, t^{1-\alpha} x(t), D_{0+}^{\alpha} x(t)\right), & x(t) \in X_{1},
\end{array}
$$


where

$$
\operatorname{dom} L_{1}=\left\{x \in X_{1}: \lim _{t \rightarrow 0^{+}} t^{1-\alpha} x(t)=x(1)\right\}, \quad \operatorname{dom} L_{2}=\left\{y \in X_{2}: \lim _{t \rightarrow 0^{+}} t^{1-\beta} y(t)=y(1)\right\} .
$$

Define the linear operator $L: \operatorname{dom} L \subset X \rightarrow Z$ and the nonlinear operator $N: X \rightarrow Z$ by

$$
\begin{aligned}
& L(x, y)=\left(L_{1} x, L_{2} y\right), \quad(x, y) \in \operatorname{dom} L, \\
& N(x, y)=\left(N_{1} y, N_{2} x\right), \quad(x, y) \in X,
\end{aligned}
$$

where

$$
\operatorname{dom} L=\left\{(x, y) \in X: x \in \operatorname{dom} L_{1}, y \in \operatorname{dom} L_{2}\right\} .
$$

Then the coupled system of BVP (1.1) is equivalent to the operator equation $L(x, y)=$ $N(x, y),(x, y) \in \operatorname{dom} L$.

Lemma 3.1 The mapping $L: \operatorname{dom} L \subset X \rightarrow Z$ is a Fredholm operator with index zero.

Proof First, we claim that the operator $L$ satisfies

$$
\begin{aligned}
& \operatorname{Ker} L=\left\{(x, y) \in \operatorname{dom} L: x(t)=c_{1} t^{\alpha-1}, y(t)=c_{2} t^{\beta-1}, c_{1}, c_{2} \in \mathbb{R}\right\} \cong \mathbb{R}^{2}, \\
& \operatorname{Im} L=\left\{(u, v) \in Z: \int_{0}^{1}(1-s)^{\alpha-1} u(s) d s=0, \int_{0}^{1}(1-s)^{\beta-1} v(s) d s=0\right\} .
\end{aligned}
$$

In fact, by Lemma 2.1, it can easily be checked that (3.1) holds. For any $(u, v) \in \operatorname{Im} L$, there exists $(x, y) \in \operatorname{dom} L$ such that $D_{0+}^{\alpha} x(t)=u(t), D_{0+}^{\beta} y(t)=v(t)$. Using Lemma 2.1 and the boundary conditions in (1.1), we find

$$
\begin{aligned}
& \int_{0}^{1}(1-s)^{\alpha-1} u(s) d s=0 \\
& \int_{0}^{1}(1-s)^{\beta-1} v(s) d s=0 .
\end{aligned}
$$

That is,

$$
\operatorname{Im} L \subset\left\{(u, v) \in Z: \int_{0}^{1}(1-s)^{\alpha-1} u(s) d s=0, \int_{0}^{1}(1-s)^{\beta-1} v(s) d s=0\right\} .
$$

Conversely, for any $(u, v) \in Z$ satisfying (3.3), take $x(t)=I_{0+}^{\alpha} u(t)$ and $y(t)=I_{0+}^{\beta} v(t)$, then we have

$$
\begin{aligned}
& \lim _{t \rightarrow 0^{+}} t^{1-\alpha} x(t)=\lim _{t \rightarrow 0^{+}} t^{1-\alpha} I_{0+}^{\alpha} u(t)=0=x(1)=\frac{1}{\Gamma(\alpha)} \int_{0}^{1}(1-s)^{\alpha-1} u(s) d s, \\
& \lim _{t \rightarrow 0^{+}} t^{1-\beta} y(t)=\lim _{t \rightarrow 0^{+}} t^{1-\beta} I_{0+}^{\beta} \nu(t)=0=y(1)=\frac{1}{\Gamma(\beta)} \int_{0}^{1}(1-s)^{\beta-1} v(s) d s,
\end{aligned}
$$


and

$$
(x, y) \in X, \quad L(x, y)=\left(L_{1} x, L_{2} y\right)=(u, v)
$$

which shows $(u, v) \in \operatorname{Im} L$. Therefore, (3.2) holds.

Second, we prove that ind $L=\operatorname{dim} \operatorname{Ker} L-c o \operatorname{dim} \operatorname{Im} L=0$. Define the linear operators $Q_{i}: Z_{1} \rightarrow Z_{1}(i=1,2)$ and $Q: Z \rightarrow Z$ by

$$
\begin{aligned}
& Q_{1} u=\alpha \int_{0}^{1}(1-s)^{\alpha-1} u(s) d s \\
& Q_{2} v=\beta \int_{0}^{1}(1-s)^{\beta-1} v(s) d s \\
& Q(u, v)=\left(Q_{1} u, Q_{2} v\right) .
\end{aligned}
$$

Evidently, $Q_{1}, Q_{2}, Q$ are continuous operators and $\operatorname{Im} L=\operatorname{Ker} Q$. For any $(u, v) \in Z$, we have

$$
\begin{aligned}
& Q_{1}^{2} u=Q_{1}\left(Q_{1} u\right)=\left(Q_{1} u\right) \alpha \int_{0}^{1}(1-s)^{\alpha-1} d s=Q_{1} u, \\
& Q_{2}^{2} v=Q_{2}\left(Q_{2} v\right)=\left(Q_{2} v\right) \beta \int_{0}^{1}(1-s)^{\beta-1} d s=Q_{2} v, \\
& Q^{2}(u, v)=Q(Q(u, v))=Q\left(Q_{1} u, Q_{2} v\right)=\left(Q_{1}^{2} u, Q_{2}^{2} v\right)=Q(u, v) .
\end{aligned}
$$

Thus, $Q$ is a continuous linear projector. For $(u, v) \in Z$, set $\left(u_{1}, v_{1}\right)=(u, v)-Q(u, v)$, then $Q\left(u_{1}, v_{1}\right)=Q(u, v)-Q^{2}(u, v)=0$, i.e., $\left(u_{1}, v_{1}\right) \in \operatorname{Ker} Q=\operatorname{Im} L$. So, $Z=\operatorname{Im} L+\operatorname{Im} Q$. Besides, for every $(u, v) \in \operatorname{Im} L \cap \operatorname{Im} Q$, we have $(u, v)=Q(u, v)=(0,0)$. Therefore, $Z=\operatorname{Im} L \oplus \operatorname{Im} Q$. Furthermore, $\operatorname{dim} \operatorname{Ker} L=\operatorname{dim} \operatorname{Im} Q=c o \operatorname{dim} \operatorname{Im} L=2$, which means $L$ is a Fredholm operator with index zero.

Lemma 3.2 Define the linear operator $K_{P}: \operatorname{Im} L \rightarrow \operatorname{dom} L \cap Y$ by

$$
K_{P}(u, v)=\left(I_{0+}^{\alpha} u, I_{0+}^{\beta} v\right), \quad(u, v) \in \operatorname{Im} L
$$

Then $K_{P}$ is the inverse of $\left.L\right|_{\operatorname{dom} L \cap Y}$ and satisfies

$$
\left\|K_{P}(u, v)\right\|_{X} \leq \Delta\|(u, v)\|_{Z} \quad \text { for all }(u, v) \in \operatorname{Im} L,
$$

where

$$
\begin{aligned}
& Y=\left\{(x, y) \in X: \lim _{t \rightarrow 0^{+}} t^{1-\alpha} x(t)=0, \lim _{t \rightarrow 0^{+}} t^{1-\beta} y(t)=0\right\} \\
& \Delta=\max \left\{1+\frac{1}{\Gamma(\alpha+1)}, 1+\frac{1}{\Gamma(\beta+1)}\right\} .
\end{aligned}
$$

Proof Define the linear operators $P_{i}: X_{i} \rightarrow X_{i}(i=1,2)$ and $P: X \rightarrow X$ by

$$
P_{1} x=\left[\lim _{t \rightarrow 0^{+}} t^{1-\alpha} x(t)\right] t^{\alpha-1}
$$




$$
\begin{aligned}
& P_{2} y=\left[\lim _{t \rightarrow 0^{+}} t^{1-\beta} y(t)\right] t^{\beta-1}, \\
& P(x, y)=\left(P_{1} x, P_{2} y\right), \quad(x, y) \in X .
\end{aligned}
$$

We first claim that $P$ is a continuous linear projector operator. In fact, for any $(x, y) \in X$, we have

$$
\begin{aligned}
& P_{1}^{2} x=P_{1}\left(P_{1} x\right)=\left[\lim _{t \rightarrow 0^{+}} t^{1-\alpha} P_{1} x(t)\right] t^{\alpha-1}=\left[\lim _{t \rightarrow 0^{+}} t^{1-\alpha} x(t)\right] t^{\alpha-1}=P_{1} x, \\
& P_{2}^{2} y=P_{2}\left(P_{2} y\right)=\left[\lim _{t \rightarrow 0^{+}} t^{1-\beta} P_{2} y(t)\right] t^{\beta-1}=\left[\lim _{t \rightarrow 0^{+}} t^{1-\beta} y(t)\right] t^{\beta-1}=P_{2} y, \\
& P^{2}(x, y)=P(P(x, y))=P\left(P_{1} x, P_{2} y\right)=\left(P_{1}^{2} x, P_{2}^{2} y\right)=\left(P_{1} x, P_{2} y\right)=P(x, y),
\end{aligned}
$$

and

$$
t^{1-\alpha} P_{1} x=\lim _{t \rightarrow 0^{+}} t^{1-\alpha} x(t), \quad t^{1-\beta} P_{2} y=\lim _{t \rightarrow 0^{+}} t^{1-\beta} y(t) .
$$

By Lemma 2.3, we also have

$$
D_{0+}^{\alpha} P_{1} x(t)=D_{0+}^{\beta} P_{2} y(t)=0 .
$$

Then

$$
\begin{aligned}
\|P(x, y)\|_{X} & =\left\|\left(P_{1} x, P_{2} y\right)\right\|_{X}=\max \left\{\left\|P_{1} x\right\|_{X_{1}},\left\|P_{2} y\right\|_{X_{2}}\right\} \\
& =\max \left\{\left|\lim _{t \rightarrow 0^{+}} t^{1-\alpha} x(t)\right|,\left|\lim _{t \rightarrow 0^{+}} t^{1-\beta} y(t)\right|\right\} .
\end{aligned}
$$

Thus, $P: X \rightarrow X$ is a bounded linear projector operator, and it is evident that $\operatorname{Im} P=$ $\operatorname{Ker} L, Y=\operatorname{Ker} P$.

Next, we show that $K_{P}=\left(\left.L\right|_{\operatorname{dom} L \cap \operatorname{Ker} P}\right)^{-1}$. In fact, for any $(u, v) \in \operatorname{Im} L$, by the definition of $K_{P}$, we can check that $K_{P}(u, v) \in \operatorname{dom} L \cap \operatorname{Ker} P$, that is, $K_{P}$ is well defined on $\operatorname{Im} L$. On the one hand, by Lemma 2.2, we have

$$
\left(L K_{P}\right)(u(t), v(t))=\left(D_{0+}^{\alpha} I_{0+}^{\alpha} u(t), D_{0+}^{\beta} I_{0+}^{\beta} v(t)\right)=(u(t), v(t)) .
$$

On the other hand, for every $(x(t), y(t)) \in \operatorname{dom} L \cap \operatorname{Ker} P$, by Lemma 2.1, we get

$$
\begin{aligned}
\left(K_{P} L\right)(x(t), y(t)) & =\left(I_{0+}^{\alpha} D_{0+}^{\alpha} x(t), I_{0+}^{\beta} D_{0+}^{\beta} y(t)\right) \\
& =\left(x(t)+c_{1} t^{\alpha-1}, y(t)+c_{2} t^{\beta-1}\right), \quad\left(c_{1}, c_{2}\right) \in \mathbb{R}^{2} .
\end{aligned}
$$

Because $\left(K_{P} L\right)(x(t), y(t)) \in \operatorname{Ker} P$ and $\left(c_{1} t^{\alpha-1}, c_{2} t^{\beta-1}\right) \in \operatorname{Ker} L=\operatorname{Im} P$, we can obtain

$$
\begin{aligned}
(0,0) & =P\left[\left(K_{P} L\right)(x(t), y(t))\right]=P\left(x(t)+c_{1} t^{\alpha-1}, y(t)+c_{2} t^{\beta-1}\right) \\
& =\left(P_{1} x(t)+c_{1} t^{\alpha-1}, P_{2} y(t)+c_{2} t^{\beta-1}\right)=\left(c_{1} t^{\alpha-1}, c_{2} t^{\beta-1}\right) .
\end{aligned}
$$


Thus, $\left(K_{P} L\right)(x(t), y(t))=(x(t), y(t))$. Therefore, $K_{P}=\left(\left.L\right|_{\operatorname{dom} L \cap \operatorname{Ker} P}\right)^{-1}$. Again by Lemma 2.2, for all $(u, v) \in \operatorname{Im} L$, we have

$$
\begin{aligned}
\left\|K_{P}(u, v)\right\|_{X} & =\max \left\{\left\|I_{0+}^{\alpha} u\right\|_{X_{1}},\left\|I_{0+}^{\beta} v\right\|_{X_{2}}\right\} \\
& =\max \left\{\left\|t^{1-\alpha} I_{0+}^{\alpha} u\right\|_{\infty}+\left\|D_{0_{+}}^{\alpha} I_{0+}^{\alpha} u\right\|_{\infty},\left\|t^{1-\beta} I_{0+}^{\beta} v\right\|_{\infty}+\left\|D_{0+}^{\beta} I_{0+}^{\beta} v\right\|_{\infty}\right\} \\
& \leq \max \left\{\left(1+\frac{1}{\Gamma(1+\alpha)}\right)\|u\|_{\infty},\left(1+\frac{1}{\Gamma(1+\beta)}\right)\|v\|_{\infty}\right\} \\
& \leq \max \left\{\Delta\|u\|_{\infty}, \Delta\|v\|_{\infty}\right\}=\Delta\|(u, v)\|_{Z^{*}}
\end{aligned}
$$

This completes the proof of Lemma 3.2.

Lemma 3.3 Assume that $\left(\mathrm{A}_{2}\right)$ holds, $\Omega \subset X$ is an open bounded subset with $\operatorname{dom} L \cap \bar{\Omega} \neq \emptyset$. Then $N$ is L-compact on $\bar{\Omega}$.

Proof Since $f, g:[0,1] \times \mathbb{R}^{2} \rightarrow \mathbb{R}$ are continuous and satisfy $\left(\mathrm{A}_{2}\right)$, we claim that $Q N(\bar{\Omega})$ and $(I-Q) N(\bar{\Omega})$ are uniformly bounded. In fact, for $\Omega$ is bounded in $X$, there exists a constant $r>0$ such that $\|(x, y)\|_{X} \leq r, \forall(x, y) \in \bar{\Omega}$, by $\left(\mathrm{A}_{2}\right)$, we have the following inequalities:

$$
\begin{aligned}
\left|N_{1} y(t)\right| & \leq\left|f\left(t, t^{1-\beta} y(t), D_{0+}^{\beta} y(t)\right)-f(t, 0,0)\right|+|f(t, 0,0)| \\
& \leq \rho+\left(p_{1}(t) t^{1-\beta}|y(t)|+q_{1}(t)\left|D_{0+}^{\beta} y(t)\right|\right) \\
& \leq \rho+\left(p_{1}+q_{1}\right) r:=r_{1}
\end{aligned}
$$

where $\rho=\sup _{t \in[0,1]} f(t, 0,0), p_{1}=\sup _{t \in[0,1]} p_{1}(t), q_{1}=\sup _{t \in[0,1]} q_{1}(t)$. In the same way, we have

$$
\left|N_{2} x(t)\right| \leq \varpi+\left(p_{2}+q_{2}\right) r:=r_{2}, \quad\left|Q_{2} N_{2} x(t)\right| \leq r_{2}
$$

where $\varpi=\sup _{t \in[0,1]} g(t, 0,0), p_{2}=\sup _{t \in[0,1]} p_{2}(t), q_{2}=\sup _{t \in[0,1]} q_{2}(t)$. So we get that

$$
\begin{aligned}
& \|Q N(x, y)\|_{Z}=\max \left\{\left\|Q_{1} N_{1} y\right\|_{Z_{1}},\left\|Q_{2} N_{2} x\right\|_{Z_{1}}\right\} \leq \max \left\{r_{1}, r_{2}\right\}, \\
& \|(I-Q) N(x, y)\|_{Z} \leq\|N(x, y)\|_{Z}+\|Q N(x, y)\|_{Z} \leq 2 \max \left\{r_{1}, r_{2}\right\} .
\end{aligned}
$$

Use of Lemma 3.2 yields

$$
\left\|K_{P}(I-Q) N(x, y)\right\|_{X} \leq \Delta\|(I-Q) N(x, y)\|_{Z} \leq 2 \Delta \max \left\{r_{1}, r_{2}\right\}
$$

From (3.4), (3.5) it follows that $Q N(\bar{\Omega}), K_{P}(I-Q) N(\bar{\Omega})$ are uniformly bounded. Now, we are going to prove that $K_{P}(I-Q) N(x, y)$ is equicontinuous for all $(x, y) \in \bar{\Omega}$. In fact, take $(x, y) \in \bar{\Omega}$ and $0 \leq t_{1}<t_{2} \leq 1$. Since $t^{\alpha}, t$ are uniformly continuous on $\left[t_{1}, t_{2}\right]$ and $f(t, u, v)$, 
$g(t, u, v)$ are uniformly continuous on $\left[t_{1}, t_{2}\right] \times[-r, r] \times[-r, r]$, we have

$$
\begin{aligned}
\left|I_{0+}^{\alpha}\left(I-Q_{1}\right) N_{1} y(t)\right|_{t=t_{1}}-\left.I_{0+}^{\alpha}\left(I-Q_{1}\right) N_{1} y(t)\right|_{t=t_{2}} \mid \\
=\frac{1}{\Gamma(\alpha)}\left|\int_{0}^{t_{1}}\left(t_{1}-s\right)^{\alpha-1}\left(I-Q_{1}\right) N_{1} y(s) d s-\int_{0}^{t_{2}}\left(t_{2}-s\right)^{\alpha-1}\left(I-Q_{1}\right) N_{1} y(s) d s\right| \\
\leq \frac{1}{\Gamma(\alpha)}\left|\int_{0}^{t_{1}}\left[\left(t_{1}-s\right)^{\alpha-1}-\left(t_{2}-s\right)^{\alpha-1}\right]\left(I-Q_{1}\right) N_{1} y(s) d s\right| \\
\quad+\frac{1}{\Gamma(\alpha)}\left|\int_{t_{1}}^{t_{2}}\left(t_{2}-s\right)^{\alpha-1}\left(I-Q_{1}\right) N_{1} y(s) d s\right| \\
\leq \frac{2 r_{1}}{\Gamma(\alpha)} \int_{0}^{t_{1}}\left[\left(t_{1}-s\right)^{\alpha-1}-\left(t_{2}-s\right)^{\alpha-1}\right] d s+\frac{2 r_{1}}{\Gamma(\alpha)} \int_{t_{1}}^{t_{2}}\left(t_{2}-s\right)^{\alpha-1} d s \\
\leq \frac{2 r_{1}}{\Gamma(\alpha+1)}\left[\left(t_{1}^{\alpha}-t_{2}^{\alpha}\right)+2\left(t_{2}-t_{1}\right)^{\alpha}\right] \rightarrow 0 \text { as } t_{1} \rightarrow t_{2},
\end{aligned}
$$

and

$$
\begin{aligned}
& \left|D_{0+}^{\alpha} I_{0+}^{\alpha}\left(I-Q_{1}\right) N_{1} y(t)\right|_{t=t_{1}}-\left.D_{0+}^{\alpha} I_{0+}^{\alpha}\left(I-Q_{1}\right) N_{1} y(t)\right|_{t=t_{2}} \mid \\
& \quad=\left|f\left(t_{1}, t_{1}^{1-\beta} y\left(t_{1}\right), D_{0+}^{\beta} y\left(t_{1}\right)\right)-f\left(t_{2}, t_{2}^{1-\beta} y\left(t_{2}\right), D_{0+}^{\beta} y\left(t_{2}\right)\right)\right| \rightarrow 0 \quad \text { as } t_{1} \rightarrow t_{2} .
\end{aligned}
$$

Similarly, it has

$$
\begin{aligned}
& \left|I_{0+}^{\beta}\left(I-Q_{2}\right) N_{2} x(t)\right|_{t=t_{1}}-\left.I_{0+}^{\beta}\left(I-Q_{2}\right) N_{2} x(t)\right|_{t=t_{2}} \mid \\
& \quad \leq \frac{2 r_{2}}{\Gamma(\beta+1)}\left[\left(t_{1}^{\beta}-t_{2}^{\beta}\right)+2\left(t_{2}-t_{1}\right)^{\beta}\right] \rightarrow 0 \quad \text { as } t_{1} \rightarrow t_{2},
\end{aligned}
$$

and

$$
\begin{aligned}
& \left|D_{0+}^{\beta} I_{0+}^{\beta}\left(I-Q_{2}\right) N_{2} x(t)\right|_{t=t_{1}}-\left.D_{0+}^{\beta} I_{0+}^{\beta}\left(I-Q_{2}\right) N_{2} x(t)\right|_{t=t_{2}} \mid \\
& \quad=\left|g\left(t_{1}, t_{1}^{1-\alpha} x\left(t_{1}\right), D_{0+}^{\alpha} x\left(t_{1}\right)\right)-g\left(t_{2}, t_{2}^{1-\alpha} x\left(t_{2}\right), D_{0+}^{\alpha} x\left(t_{2}\right)\right)\right| \rightarrow 0 \quad \text { as } t_{1} \rightarrow t_{2} .
\end{aligned}
$$

To summarize, we can conclude that $\left\{K_{P}(I-Q) N(x, y):(x, y) \in \bar{\Omega}\right\}$ is equicontinuous. By the Ascoli-Arzelà theorem, it is immediate that $K_{P}(I-Q) N: \bar{\Omega} \rightarrow X$ is compact. Using a similar argument, we can also get $N$ is $L$-compact if condition $\left(\mathrm{A}_{1}\right)$ holds.

In what follows, we shall give several existence results for BVP (1.1). For simplicity of presentation, we let

$$
\begin{array}{lll}
\rho_{1}=\frac{\omega_{2}+\omega_{1} \eta_{2}}{1-\eta_{1} \eta_{2}}, & \rho_{2}=\frac{\gamma_{2}}{1-\eta_{1} \eta_{2}}, & \rho_{3}=\frac{\gamma_{1} \eta_{2}}{1-\eta_{1} \eta_{2}}, \\
\sigma_{1}=\frac{\omega_{1}+\omega_{2} \eta_{1}}{1-\eta_{1} \eta_{2}}, & \sigma_{2}=\frac{\gamma_{2} \eta_{1}}{1-\eta_{1} \eta_{2}}, & \sigma_{3}=\frac{\gamma_{1}}{1-\eta_{1} \eta_{2}},
\end{array}
$$

where $\gamma_{i}=\max _{t \in[0,1]}|\gamma(t)|, \eta_{i}=\max _{t \in[0,1]}|\gamma(t)|, \omega_{i}=\max _{t \in[0,1]}|\gamma(t)|, i=1,2$. First, we suppose that the following conditions are satisfied:

$\left(\mathrm{A}_{4}\right)$ For $(x, y) \in \operatorname{dom} L$, there exist constants $B_{i}>0, i=1,2$, such that, for all $t \in[0,1]$, if either $\left|t^{1-\alpha} x(t)\right|>B_{1}$ or $\left|t^{1-\beta} y(t)\right|>B_{2}$, then $Q N(x, y) \neq(0,0)$. 
( $\left.\mathrm{A}_{5}\right)$ For $\left(c_{1} t^{\alpha-1}, c_{2} t^{\beta-1}\right) \in \operatorname{Ker} L$, there exist constants $G_{i}>0, i=1,2$, such that for any $\left(c_{1}, c_{2}\right) \in \mathbb{R}^{2}$ satisfying either

$$
\begin{cases}c_{1} N_{2}\left(c_{1} t^{\alpha-1}\right)>0, & \text { if }\left|c_{1}\right|>G_{1}, \\ c_{2} N_{1}\left(c_{2} t^{\beta-1}\right)>0, & \text { if }\left|c_{2}\right|>G_{2},\end{cases}
$$

or

$$
\begin{cases}c_{1} N_{2}\left(c_{1} t^{\alpha-1}\right)<0, & \text { if }\left|c_{1}\right|>G_{1}, \\ c_{2} N_{1}\left(c_{2} t^{\beta-1}\right)<0, & \text { if }\left|c_{2}\right|>G_{2} .\end{cases}
$$

Lemma 3.4 Let $\left(\mathrm{A}_{1}\right)$ and $\left(\mathrm{A}_{4}\right)$ hold, set

$$
\left\{\Omega_{1}=(x, y) \in \operatorname{dom} L \backslash \operatorname{Ker} L: L(x, y)=\lambda N(x, y), \lambda \in(0,1)\right\} .
$$

Then $\Omega_{1}$ is bounded provided that

$$
\begin{aligned}
& \eta_{1} \eta_{2}<1, \quad \Gamma(\alpha+1)>2 \sigma_{2}, \quad \Gamma(\beta+1)>2 \rho_{3}, \\
& \left(\Gamma(\alpha+1)-2 \sigma_{2}\right)\left(\Gamma(\beta+1)-2 \rho_{3}\right)>4 \rho_{2} \sigma_{3},
\end{aligned}
$$

or

$$
\begin{array}{ll}
\frac{2}{\Gamma(\alpha+1)}\left(\gamma_{1}+\eta_{1}\right)+\Delta\left(\gamma_{2}+\eta_{2}\right)<1, & \left(\frac{2}{\Gamma(\alpha+1)}+\Delta\right)\left(\gamma_{1}+\eta_{1}\right)<1, \\
\frac{2}{\Gamma(\beta+1)}\left(\gamma_{2}+\eta_{2}\right)+\Delta\left(\gamma_{1}+\eta_{1}\right)<1, & \left(\frac{2}{\Gamma(\beta+1)}+\Delta\right)\left(\gamma_{2}+\eta_{2}\right)<1 .
\end{array}
$$

Proof For $(x, y) \in \Omega_{1}$, we have $N(x, y) \in \operatorname{Im} L=\operatorname{Ker} Q$. Then $Q N(x, y)=(0,0)$. On the one hand, according to hypothesis $\left(\mathrm{A}_{4}\right)$, it follows that

$$
\left|t_{2}^{1-\alpha} x\left(t_{2}\right)\right| \leq B_{1}, \quad\left|t_{1}^{1-\beta} y\left(t_{1}\right)\right| \leq B_{2} .
$$

Since

$$
I_{0_{+}}^{\alpha} D_{0_{+}}^{\alpha} x(t)=x(t)+c_{1} t^{\alpha-1}, \quad c_{1} \in \mathbb{R}, \quad I_{0_{+}}^{\beta} D_{0+}^{\beta} y(t)=y(t)+c_{2} t^{\beta-1}, \quad c_{2} \in \mathbb{R} .
$$

By substituting (3.10) into (3.11), we obtain

$$
c_{1}=\left.t^{1-\alpha} I_{0+}^{\alpha} D_{0+}^{\alpha} x(t)\right|_{t=t_{2}}-t_{2}^{1-\alpha} x\left(t_{2}\right), \quad c_{2}=\left.t^{1-\beta} I_{0+}^{\beta} D_{0+}^{\beta} y(t)\right|_{t=t_{1}}-t_{1}^{1-\beta} y\left(t_{1}\right) .
$$

From (3.10), (3.11), and (3.12) we have

$$
\left|t^{1-\alpha} x(t)\right| \leq B_{1}+\frac{2}{\Gamma(\alpha+1)}\left\|D_{0+}^{\alpha} x\right\|_{\infty}, \quad\left|t^{1-\beta} y(t)\right| \leq B_{2}+\frac{2}{\Gamma(\beta+1)}\left\|D_{0+}^{\beta} y\right\|_{\infty} .
$$

On the other hand, by $\left(\mathrm{A}_{1}\right)$, we have

$$
\begin{aligned}
\left|D_{0+}^{\alpha} x\right| & =\left|f\left(t, t^{1-\beta} y, D_{0+}^{\beta} y\right)\right| \leq \gamma_{1}(t)\left|t^{1-\beta} y\right|+\eta_{1}(t)\left|D_{0+}^{\beta} y\right|+w_{1}(t) \\
& \leq \gamma_{1}\left|t^{1-\beta} y\right|+\eta_{1}\left|D_{0+}^{\beta} y\right|+\omega_{1},
\end{aligned}
$$




$$
\begin{aligned}
\left|D_{0+}^{\beta} y\right| & =\left|g\left(t, t^{1-\alpha} x, D_{0+}^{\alpha} x\right)\right| \leq \gamma_{2}(t)\left|t^{1-\alpha} x\right|+\eta_{2}(t)\left|D_{0+}^{\alpha} x\right|+w_{2}(t) \\
& \leq \gamma_{2}\left|t^{1-\alpha} x\right|+\eta_{2}\left|D_{0+}^{\alpha} x\right|+\omega_{2} .
\end{aligned}
$$

We now estimate $\Omega_{1}$ is bounded under conditions (3.8) and (3.9), respectively.

First. We show that $\Omega_{1}$ is bounded if condition (3.8) holds. In fact, (3.14) and (3.15) imply that

$$
\left|D_{0+}^{\alpha} x\right| \leq \sigma_{1}+\sigma_{2}\left|t^{1-\alpha} x\right|+\sigma_{3}\left|t^{1-\beta} y\right|, \quad\left|D_{0+}^{\beta} y\right| \leq \rho_{1}+\rho_{2}\left|t^{1-\alpha} x\right|+\rho_{3}\left|t^{1-\beta} y\right| .
$$

If we plug (3.16) back into (3.13), we get

$$
\begin{aligned}
& \left|t^{1-\alpha} x\right| \leq B_{1}+\frac{2}{\Gamma(\alpha+1)}\left(\sigma_{1}+\sigma_{2}\left|t^{1-\alpha} x\right|+\sigma_{3}\left|t^{1-\beta} y\right|\right), \\
& \left|t^{1-\beta} y\right| \leq B_{2}+\frac{2}{\Gamma(\beta+1)}\left(\rho_{1}+\rho_{2}\left|t^{1-\alpha} x\right|+\rho_{3}\left|t^{1-\beta} y\right|\right) .
\end{aligned}
$$

So that

$$
\begin{aligned}
& \left|t^{1-\alpha} x\right| \leq \frac{B_{1} \Gamma(\alpha+1)+2 \sigma_{1}}{\Gamma(\alpha+1)-2 \sigma_{2}}+\frac{2 \sigma_{3}}{\Gamma(\alpha+1)-2 \sigma_{2}}\left|t^{1-\beta} y\right|:=\ell_{1}+\ell_{2}\left|t^{1-\beta} y\right|, \\
& \left|t^{1-\beta} y\right| \leq \frac{B_{2} \Gamma(\beta+1)+2 \rho_{1}}{\Gamma(\beta+1)-2 \rho_{3}}+\frac{2 \rho_{2}}{\Gamma(\beta+1)-2 \rho_{3}}\left|t^{1-\alpha} x\right|:=\delta_{1}+\delta_{2}\left|t^{1-\alpha} x\right| .
\end{aligned}
$$

It follows from (3.17) and (3.18) that

$$
\left|t^{1-\beta} y\right| \leq \frac{\delta_{1}+\delta_{2} \ell_{1}}{1-\delta_{2} \ell_{2}}:=\tau, \quad\left|t^{1-\alpha} x\right| \leq \ell_{1}+\ell_{2} \tau
$$

Substituting (3.19) into (3.16), we obtain

$$
\left|D_{0+}^{\alpha} x\right| \leq \sigma_{1}+\sigma_{2}\left(\ell_{1}+\ell_{2} \tau\right)+\sigma_{3} \tau, \quad\left|D_{0+}^{\beta} y\right| \leq \rho_{1}+\rho_{2}\left(\ell_{1}+\ell_{2} \tau\right)+\rho_{3} \tau .
$$

Thus, $\Omega_{1}$ is bounded.

Second. We prove that $\Omega_{1}$ is bounded under condition (3.9). In such a case, by Lemma 3.2, one has

$$
\begin{aligned}
\|(I-P)(x, y)\|_{X} & =\left\|K_{P} L(I-P)(x, y)\right\|_{X} \leq \Delta\|L(x, y)\|_{Z} \\
& =\Delta \max \left\{\left\|D_{0+}^{\alpha} x\right\|_{\infty},\left\|D_{0+}^{\beta} y\right\|_{\infty}\right\} .
\end{aligned}
$$

Therefore, from (3.13)-(3.15), we can derive that

$$
\begin{aligned}
\|(x, y)\|_{X} \leq & \|P(x, y)\|_{X}+\|(I-P)(x, y)\|_{X} \\
\leq & \max \left\{\left\|t^{1-\beta} y\right\|_{\infty},\left\|t^{1-\alpha} x\right\|_{\infty}\right\}+\Delta \max \left\{\left\|D_{0+}^{\alpha} x\right\|_{\infty},\left\|D_{0+}^{\beta} y\right\|_{\infty}\right\} \\
\leq & \max \left\{B_{2}+\frac{2}{\Gamma(\beta+1)}\left\|D_{0+}^{\beta} y\right\|_{\infty}, B_{1}+\frac{2}{\Gamma(\alpha+1)}\left\|D_{0+}^{\alpha} x\right\|_{\infty}\right\} \\
& +\Delta \max \left\{\left\|D_{0+}^{\alpha} x\right\|_{\infty},\left\|D_{0+}^{\beta} y\right\|_{\infty}\right\}
\end{aligned}
$$




$$
\begin{aligned}
\leq & \max \left\{B_{1}+\frac{2}{\Gamma(\alpha+1)}\left\|D_{0+}^{\alpha} x\right\|_{\infty}+\Delta\left\|D_{0+}^{\beta} y\right\|_{\infty},\right. \\
& B_{1}+\left(\frac{2}{\Gamma(\alpha+1)}+\Delta\right)\left\|D_{0+}^{\alpha} x\right\|_{\infty}, \\
& B_{2}+\frac{2}{\Gamma(\beta+1)}\left\|D_{0+}^{\beta} y\right\|_{\infty}+\Delta\left\|D_{0+}^{\alpha} x\right\|_{\infty}, \\
& \left.B_{2}+\left(\frac{2}{\Gamma(\beta+1)}+\Delta\right)\left\|D_{0+}^{\beta} y\right\|_{\infty}\right\} .
\end{aligned}
$$

Next, we separate the proof into four cases.

Case 1. $\|(x, y)\|_{X} \leq B_{1}+\frac{2}{\Gamma(\alpha+1)}\left\|D_{0_{+}}^{\alpha} x\right\|_{\infty}+\Delta\left\|D_{0_{+}}^{\beta} y\right\|_{\infty}$ because

$$
\max \left\{\left\|t^{1-\alpha} x\right\|_{\infty},\left\|D_{0_{+}}^{\alpha} x\right\|_{\infty},\left\|t^{1-\beta} y\right\|_{\infty},\left\|D_{0+}^{\beta} y\right\|_{\infty}\right\} \leq\|(x, y)\|_{X} .
$$

By (3.14) and (3.15), one gets

$$
\begin{aligned}
\|(x, y)\|_{X} & \leq B_{1}+\frac{2}{\Gamma(\alpha+1)}\left\|D_{0+}^{\alpha} x\right\|_{\infty}+\Delta\left\|D_{0+}^{\beta} y\right\|_{\infty} \\
& \leq B_{1}+\frac{2}{\Gamma(\alpha+1)}\left[\left(\gamma_{1}+\eta_{1}\right)\|(x, y)\|_{X}+\omega_{1}\right]+\Delta\left[\left(\gamma_{2}+\eta_{2}\right)\|(x, y)\|_{X}+\omega_{2}\right],
\end{aligned}
$$

that is,

$$
\|(x, y)\|_{X} \leq \frac{B_{1}+\left(2 \omega_{1} / \Gamma(\alpha+1)\right)+\Delta \omega_{2}}{1-\left[\left(2\left(\gamma_{1}+\eta_{1}\right) / \Gamma(\alpha+1)\right)+\Delta\left(\gamma_{2}+\eta_{2}\right)\right]} .
$$

Case 2. $\|(x, y)\|_{X} \leq B_{1}+\left(\frac{2}{\Gamma(\alpha+1)}+\Delta\right)\left\|D_{0+}^{\alpha} x\right\|_{\infty}$. By (3.14) and (3.20), we have

$$
\begin{aligned}
\|(x, y)\|_{X} & \leq B_{1}+\left(\frac{2}{\Gamma(\alpha+1)}+\Delta\right)\left\|D_{0+}^{\alpha} x\right\|_{\infty} \\
& \leq B_{1}+\left(\frac{2}{\Gamma(\alpha+1)}+\Delta\right)\left[\left(\gamma_{1}+\eta_{1}\right)\|(x, y)\|_{X}+\omega_{1}\right],
\end{aligned}
$$

which implies that

$$
\|(x, y)\|_{X} \leq \frac{B_{1}+((2 / \Gamma(\alpha+1))+\Delta) \omega_{1}}{1-((2 / \Gamma(\alpha+1))+\Delta)\left(\gamma_{1}+\eta_{1}\right)} .
$$

Case 3. $\|(x, y)\|_{X} \leq B_{2}+\frac{2}{\Gamma(\beta+1)}\left\|D_{0+}^{\beta} y\right\|_{\infty}+\Delta\left\|D_{0_{+}}^{\alpha} x\right\|_{\infty}$. Using a similar proof as that in Case 1, we can get

$$
\|(x, y)\|_{X} \leq \frac{B_{2}+\left(2 \omega_{2} / \Gamma(\beta+1)\right)+\Delta \omega_{1}}{1-\left[\left(2\left(\gamma_{2}+\eta_{2}\right) / \Gamma(\beta+1)\right)+\Delta\left(\gamma_{1}+\eta_{1}\right)\right]} .
$$

Case 4. $\|(x, y)\|_{X} \leq B_{2}+\left(\frac{2}{\Gamma(\beta+1)}+\Delta\right)\left\|D_{0+}^{\beta} y\right\|_{\infty}$. By applying a method similar to Case 2, we can obtain

$$
\|(x, y)\|_{X} \leq \frac{B_{2}+((2 / \Gamma(\beta+1))+\Delta) \omega_{2}}{1-((2 / \Gamma(\beta+1))+\Delta)\left(\gamma_{2}+\eta_{2}\right)} .
$$

To summarize, $\Omega_{1}$ is bounded and the proof is completed. 
Remark 3.1 If $\alpha=\beta$, then condition (3.8) can be derived by (3.9).

In fact, from

$$
\frac{2}{\Gamma(\alpha+1)}\left(\gamma_{1}+\eta_{1}\right)+\Delta\left(\gamma_{2}+\eta_{2}\right)<1, \quad \frac{2}{\Gamma(\beta+1)}\left(\gamma_{2}+\eta_{2}\right)+\Delta\left(\gamma_{1}+\eta_{1}\right)<1,
$$

we can obtain

$$
\gamma_{1}+\eta_{1}<1, \quad \gamma_{2}+\eta_{2}<1, \quad 2 \eta_{1}<\Gamma(\alpha+1), \quad 2 \eta_{2}<\Gamma(\beta+1) .
$$

On the other hand, by

$$
\left(\frac{2}{\Gamma(\alpha+1)}+\Delta\right)\left(\gamma_{1}+\eta_{1}\right)<1, \quad\left(\frac{2}{\Gamma(\beta+1)}+\Delta\right)\left(\gamma_{2}+\eta_{2}\right)<1
$$

we have

$$
\left(\frac{2}{\Gamma(\alpha+1)}+1\right)\left(\gamma_{1}+\eta_{1}\right)<1, \quad\left(\frac{2}{\Gamma(\beta+1)}+1\right)\left(\gamma_{2}+\eta_{2}\right)<1
$$

Using the fact $\alpha=\beta$, we also have

$$
\left(\frac{2}{\Gamma(\beta+1)}+1\right)\left(\gamma_{1}+\eta_{1}\right)<1, \quad\left(\frac{2}{\Gamma(\alpha+1)}+1\right)\left(\gamma_{2}+\eta_{2}\right)<1
$$

Then it follows that

$$
(2+\Gamma(\alpha+1)) \zeta<\Gamma(\alpha+1), \quad(2+\Gamma(\beta+1)) \zeta<\Gamma(\beta+1)
$$

where $\zeta=\max \left\{\gamma_{1}, \eta_{1}, \gamma_{2}, \eta_{2}\right\}$. From (3.21) and (3.22), we have

$$
\begin{aligned}
& \Gamma(\alpha+1) \eta_{1} \eta_{2}+2 \gamma_{2} \eta_{1}=\eta_{1}\left(\Gamma(\alpha+1) \eta_{2}+2 \gamma_{2}\right)<2 \eta_{1}\left(\eta_{2}+\gamma_{2}\right)<2 \eta_{1}<\Gamma(\alpha+1), \\
& \Gamma(\beta+1) \eta_{1} \eta_{2}+2 \gamma_{1} \eta_{2}=\eta_{2}\left(\Gamma(\beta+1) \eta_{1}+2 \gamma_{1}\right)<2 \eta_{2}\left(\eta_{1}+\gamma_{1}\right)<2 \eta_{2}<\Gamma(\beta+1), \\
& 2 \sigma_{2}+2 \rho_{2}=\frac{2 \gamma_{2} \eta_{1}}{1-\eta_{1} \eta_{2}}+\frac{2 \gamma_{2}}{1-\eta_{1} \eta_{2}}=\frac{2 \gamma_{2}\left(1+\eta_{1}\right)}{1-\eta_{1} \eta_{2}}<\frac{2 \zeta(1+\zeta)}{1-\zeta^{2}}=\frac{2 \zeta}{1-\zeta}<\Gamma(\alpha+1), \\
& 2 \sigma_{3}+2 \rho_{3}=\frac{2 \gamma_{1}}{1-\eta_{1} \eta_{2}}+\frac{2 \gamma_{1} \eta_{2}}{1-\eta_{1} \eta_{2}}=\frac{2 \gamma_{1}\left(1+\eta_{2}\right)}{1-\eta_{1} \eta_{2}}<\frac{2 \zeta(1+\zeta)}{1-\zeta^{2}}=\frac{2 \zeta}{1-\zeta}<\Gamma(\beta+1) .
\end{aligned}
$$

According to the above inequalities, it follows (3.8) holds.

Lemma 3.5 Let $\left(\mathrm{A}_{4}\right)$ hold, set

$$
\Omega_{2}=\{(x, y) \in \operatorname{Ker} L: N(x, y) \in \operatorname{Im} L\} .
$$

Then $\Omega_{2}$ is bounded.

Proof For $(x, y) \in \operatorname{Ker} L$, then we can write $x=c_{1} t^{\alpha-1}, y=c_{2} t^{\beta-1},\left(c_{1}, c_{2}\right) \in \mathbb{R}^{2}$, and $N\left(c_{1} t^{\alpha-1}, c_{2} t^{\beta-1}\right) \in \operatorname{Im} L=\operatorname{Ker} Q$, that is, $Q N\left(c_{1} t^{\alpha-1}, c_{2} t^{\beta-1}\right)=(0,0)$. By $\left(\mathrm{A}_{4}\right)$, there exist 
$t_{3}, t_{4} \in[0,1]$ such that $\left|t_{3}^{1-\alpha} x\left(t_{3}\right)\right|=\left|c_{1}\right| \leq B_{1},\left|t_{4}^{1-\beta} y\left(t_{4}\right)\right|=\left|c_{2}\right| \leq B_{2}$. Therefore,

$$
\|(x, y)\|_{X}=\max \left\{\|x\|_{X_{1}},\|y\|_{X_{2}}\right\}=\max \left\{\left|c_{1}\right|,\left|c_{2}\right|\right\} \leq \max \left\{B_{1}, B_{2}\right\} .
$$

The proof is completed.

Lemma 3.6 Let $\left(\mathrm{A}_{5}\right)$ hold, set

$$
\Omega_{3}=\{(x, y) \in \operatorname{Ker} L: \vartheta \lambda J(x, y)+(1-\lambda) Q N(x, y)=(0,0), \lambda \in[0,1]\} .
$$

Then $\Omega_{3}$ is bounded, where

$$
\vartheta= \begin{cases}1, & \text { if (3.6) holds, } \\ -1, & \text { if (3.7) holds }\end{cases}
$$

and $J: \operatorname{Ker} L \rightarrow \operatorname{Im} Q$ is the linear isomorphism given by

$$
J\left(c_{1} t^{\alpha-1}, c_{2} t^{\beta-1}\right)=\left(c_{2}, c_{1}\right), \quad \forall c_{1}, c_{2} \in \mathbb{R}^{2}, t \in[0,1] .
$$

Proof Without loss of generality, we suppose that (3.7) holds, then for $(x, y) \in \Omega_{3}$, we have

$$
\begin{aligned}
& \lambda c_{2}=(1-\lambda) \alpha \int_{0}^{1}(1-s)^{\alpha-1} f\left(s, c_{2}, 0\right) d s, \\
& \lambda c_{1}=(1-\lambda) \beta \int_{0}^{1}(1-s)^{\beta-1} g\left(s, c_{1}, 0\right) d s .
\end{aligned}
$$

By the preceding lemma, it suffices to show that $\left|c_{1}\right|,\left|c_{2}\right|$ are bounded. In fact, if $\lambda=1$, then $c_{1}=c_{2}=0$. Otherwise, for $\lambda \in[0,1)$, we get

$$
\begin{aligned}
& 0 \leq \lambda c_{2}^{2}=(1-\lambda) \alpha \int_{0}^{1}(1-s)^{\alpha-1} c_{2} f\left(s, c_{2}, 0\right) d s, \\
& 0 \leq \lambda c_{1}^{2}=(1-\lambda) \beta \int_{0}^{1}(1-s)^{\beta-1} c_{1} g\left(s, c_{1}, 0\right) d s .
\end{aligned}
$$

If $\left|c_{1}\right|>G_{1}$ or $\left|c_{2}\right|>G_{2}$, by (3.7), it is easy to verify that at least one of the above equations is not true. Therefore, $\left|c_{1}\right|,\left|c_{2}\right|$ are bounded, which completes the proof of Lemma 3.6.

Lemma 3.7 Let $\left(\mathrm{A}_{2}\right)$ hold, set

$$
\Omega_{4}=\{(x, y) \in \operatorname{dom} L \backslash \operatorname{Ker} L: L(x, y)-N(x, y)=-\lambda(L(x, y)+N(-x,-y)), \lambda \in(0,1]\} .
$$

Then $\Omega_{4}$ is bounded provided that

$$
\Gamma(\alpha+1) \Gamma(\beta+1)>(1+\Gamma(\alpha+1))(1+\Gamma(\beta+1))\left(p_{1}+q_{1}\right)\left(p_{2}+q_{2}\right),
$$

where $p_{i}=\sup _{t \in[0,1]} p_{i}(t), q_{i}=\sup _{t \in[0,1]} q_{i}(t), i=1,2$. 
Proof For $(x, y) \in \Omega_{4}$, we have

$$
L(x, y)=\frac{1}{1+\lambda} N(x, y)-\frac{\lambda}{1+\lambda} N(-x,-y),
$$

that is,

$$
\begin{aligned}
& L_{1} x=\frac{1}{1+\lambda} N_{1} y-\frac{\lambda}{1+\lambda} N_{1}(-y), \\
& L_{2} y=\frac{1}{1+\lambda} N_{2} x-\frac{\lambda}{1+\lambda} N_{2}(-x) .
\end{aligned}
$$

From (3.24) it follows that, for any $t \in[0,1]$,

$$
\begin{aligned}
\left|L_{1} x\right|= & \left|D_{0+}^{\alpha} x\right| \leq \frac{1}{1+\lambda}\left|N_{1} y\right|+\frac{\lambda}{1+\lambda}\left|N_{1}(-y)\right| \\
= & \frac{1}{1+\lambda}\left|f\left(t, t^{1-\beta} y(t), D_{0+}^{\beta} y(t)\right)\right|+\frac{\lambda}{1+\lambda}\left|f\left(t,-t^{1-\beta} y(t),-D_{0+}^{\beta} y(t)\right)\right| \\
\leq & \frac{1}{1+\lambda}\left[\left|f\left(t, t^{1-\beta} y(t), D_{0+}^{\beta} y(t)\right)-f(t, 0,0)\right|+\rho\right] \\
& +\frac{\lambda}{1+\lambda}\left[\left|f\left(t,-t^{1-\beta} y(t),-D_{0+}^{\beta} y(t)\right)-f(t, 0,0)\right|+\rho\right] \\
\leq & \rho+p_{1}\left|t^{1-\beta} y(t)\right|+q_{1}\left|D_{0+}^{\beta} y(t)\right| \\
\leq & \rho+p_{1}\left\|t^{1-\beta} y\right\|_{\infty}+q_{1}\left\|D_{0+}^{\beta} y\right\|_{\infty},
\end{aligned}
$$

and

$$
\begin{aligned}
\left|t^{1-\alpha} x(t)\right|= & \frac{1}{1+\lambda}\left|t^{1-\alpha} I_{0+}^{\alpha} N_{1} y-\lambda t^{1-\alpha} I_{0+}^{\alpha} N_{1}(-y)\right| \\
\leq & \frac{\rho}{\Gamma(\alpha+1)}+\frac{t^{1-\alpha}}{(1+\lambda) \Gamma(\alpha)} \int_{0}^{t}(t-s)^{\alpha-1}\left|f\left(s, s^{1-\beta} y(s), D_{0+}^{\beta} y(s)\right)-f(t, 0,0)\right| d s \\
& +\frac{\lambda t^{1-\alpha}}{(1+\lambda) \Gamma(\alpha)} \int_{0}^{t}(t-s)^{\alpha-1}\left|f\left(s,-s^{1-\beta} y(s),-D_{0+}^{\beta} y(s)\right)-f(t, 0,0)\right| d s \\
\leq & \frac{1}{\Gamma(\alpha+1)}\left[p_{1}\left\|t^{1-\beta} y\right\|_{\infty}+q_{1}\left\|D_{0+}^{\beta} y\right\|_{\infty}\right]+\frac{\rho}{\Gamma(\alpha+1)} .
\end{aligned}
$$

Taking account of (3.26) and (3.27), we derive

$$
\begin{aligned}
\left\|D_{0+}^{\alpha} x\right\|_{\infty} & \leq \rho+p_{1}\left\|t^{1-\beta} y\right\|_{\infty}+q_{1}\left\|D_{0+}^{\beta} y\right\|_{\infty} \\
\left\|t^{1-\alpha} x\right\|_{\infty} & \leq \frac{1}{\Gamma(\alpha+1)}\left[p_{1}\left\|t^{1-\beta} y\right\|_{\infty}+q_{1}\left\|D_{0+}^{\beta} y\right\|_{\infty}+\rho\right] .
\end{aligned}
$$

Similarly, by (3.25), it can be shown that, for any $t \in[0,1]$,

$$
\begin{aligned}
& \left\|D_{0+}^{\beta} y\right\|_{\infty} \leq \varpi+p_{2}\left\|t^{1-\alpha} x\right\|_{\infty}+q_{2}\left\|D_{0+}^{\alpha} x\right\|_{\infty} \\
& \left\|t^{1-\beta} y\right\|_{\infty} \leq \frac{1}{\Gamma(\beta+1)}\left[p_{2}\left\|t^{1-\alpha} x\right\|_{\infty}+q_{2}\left\|D_{0_{+}}^{\alpha} x\right\|_{\infty}+\varpi\right] .
\end{aligned}
$$


According to (3.28)-(3.31), we get

$$
\begin{aligned}
\|x\|_{X_{1}} & \leq \frac{1+\Gamma(\alpha+1)}{\Gamma(\alpha+1)}\left[p_{1}\left\|t^{1-\beta} y\right\|_{\infty}+q_{1}\left\|D_{0+}^{\beta} y\right\|_{\infty}+\rho\right] \\
& \leq \frac{1+\Gamma(\alpha+1)}{\Gamma(\alpha+1)}\left[\left(p_{1}+q_{1}\right)\|y\|_{X_{2}}+\rho\right], \\
\|y\|_{X_{2}} & \leq \frac{1+\Gamma(\beta+1)}{\Gamma(\beta+1)}\left[p_{2}\left\|t^{1-\alpha} x\right\|_{\infty}+q_{2}\left\|D_{0+}^{\alpha} x\right\|_{\infty}+\varpi\right] \\
& \leq \frac{1+\Gamma(\beta+1)}{\Gamma(\beta+1)}\left[\left(p_{2}+q_{2}\right)\|x\|_{X_{1}}+\varpi\right] .
\end{aligned}
$$

Now, by using (3.32) and (3.33), we obtain

$$
\begin{aligned}
\|y\|_{X_{2}} & \leq \frac{(1+\Gamma(\alpha+1))(1+\Gamma(\beta+1))\left(p_{2}+q_{2}\right) \rho+\Gamma(\alpha+1)(1+\Gamma(\beta+1)) \varpi}{\Gamma(\alpha+1) \Gamma(\beta+1)-(1+\Gamma(\alpha+1))(1+\Gamma(\beta+1))\left(p_{1}+q_{1}\right)\left(p_{2}+q_{2}\right)}:=m_{1} \\
\|x\|_{X_{1}} & \leq \frac{1+\Gamma(\alpha+1)}{\Gamma(\alpha+1)}\left[\left(p_{1}+q_{1}\right) m_{1}+\rho\right]:=m_{2} .
\end{aligned}
$$

So we get that

$$
\begin{aligned}
\|(x, y)\|_{X} & =\max \left\{\|x\|_{X_{1}},\|y\|_{X_{2}}\right\} \leq \max \left\{m_{1}, m_{2}\right\} \\
& =\frac{1}{2}\left(m_{1}+m_{2}+\left|m_{1}-m_{2}\right|\right):=m .
\end{aligned}
$$

This completes the proof of the lemma.

Theorem 3.1 Assume that $\left(\mathrm{A}_{1}\right),\left(\mathrm{A}_{4}\right),\left(\mathrm{A}_{5}\right)$, and (3.8) hold or $\left(\mathrm{A}_{1}\right),\left(\mathrm{A}_{4}\right),\left(\mathrm{A}_{5}\right)$, and (3.9) hold. Then BVP (1.1) has at least one solution in $X$.

Proof Set $\Omega$ be a bounded open set of $X$ such that $\bigcup_{i=1}^{3} \bar{\Omega}_{i} \subset \Omega$. By Lemma 3.3, $N$ is $L$ compact on $\bar{\Omega}$. Lemmas 3.4 and 3.5 imply that (i) and (ii) of Theorem 2.1 are satisfied. In order to achieve the thesis, we have to prove that condition (iii) of Theorem 2.1 holds. Define the homotopy mapping as follows:

$$
H((x, y), \lambda)=\vartheta \lambda J(x, y)+(1-\lambda) Q N(x, y)
$$

By Lemma 3.6, we get $H((x, y), \lambda) \neq(0,0)$ for all $(x, y) \in \operatorname{Ker} L \cap \partial \Omega$. Using the homotopy invariance of the topological degree,

$$
\begin{aligned}
\operatorname{deg}\left\{\left.Q N\right|_{\operatorname{Ker} L}, \Omega \cap \operatorname{Ker} L,(0,0)\right\} & =\operatorname{deg}\{H(\cdot, 0), \Omega \cap \operatorname{Ker} L,(0,0)\} \\
& =\operatorname{deg}\{H(\cdot, 1), \Omega \cap \operatorname{Ker} L,(0,0)\} \\
& =\operatorname{deg}\{\vartheta J, \Omega \cap \operatorname{Ker} L,(0,0)\} \neq 0 .
\end{aligned}
$$

Then, by Theorem 2.1, BVP (1.1) has at least one solution in $X$. Thus the theorem is proved.

Theorem 3.2 If $\left(\mathrm{A}_{2}\right)$ and (3.23) hold, then BVP (1.1) has at least one solution in $X$. 
Proof Set $\Omega=\left\{(x, y) \in X:\|(x, y)\|_{X}<m+1\right\}$. Obviously, $\Omega$ is symmetric with $(0,0) \in \Omega$ and $X \cap \bar{\Omega} \neq \emptyset$. By Lemma 3.7, we get, for every $(x, y) \in \partial \Omega$ and $\lambda \in(0,1]$,

$$
L(x, y)-N(x, y) \neq-\lambda(L(x, y)+N(-x,-y)) \text {, }
$$

which together with Theorem 2.2 yields that problem (1.1) has at least one solution in $X$.

Theorem 3.3 If $\left(\mathrm{A}_{2}\right),\left(\mathrm{A}_{3}\right)$, and (3.23) hold, then BVP (1.1) has exactly one solution in $X$ provided that

$$
\begin{gathered}
\max \left\{(\kappa+\Delta)\left(p_{1}+q_{1}\right), \kappa\left(p_{1}+q_{1}\right)+\Delta\left(p_{2}+q_{2}\right),\right. \\
\left.\mu\left(p_{2}+q_{2}\right)+\Delta\left(p_{1}+q_{1}\right),(\mu+\Delta)\left(p_{2}+q_{2}\right)\right\}<1,
\end{gathered}
$$

where

$$
\kappa=\left(\frac{2}{\Gamma(\alpha+1)}+\frac{d}{c}\right), \quad \mu=\left(\frac{2}{\Gamma(\beta+1)}+\frac{b}{a}\right) .
$$

Proof By Theorem 3.2, we obtain that BVP (1.1) has at least one solution in $X$. Now, we prove the uniqueness result. Suppose that BVP (1.1) has two solutions $\left(x_{1}, y_{1}\right),\left(x_{2}, y_{2}\right) \in$ $\operatorname{dom} L$. Then, for $i=1,2$, we have

$$
\begin{aligned}
& D_{0+}^{\alpha} x_{i}(t)=f\left(t, t^{1-\beta} y_{i}(t), D_{0+}^{\beta} y_{i}(t)\right), \\
& D_{0+}^{\beta} y_{i}(t)=g\left(t, t^{1-\alpha} x_{i}(t), D_{0+}^{\alpha} x_{i}(t)\right),
\end{aligned}
$$

and

$$
\lim _{t \rightarrow 0^{+}} t^{1-\alpha} x_{i}(t)=x_{i}(1), \quad \lim _{t \rightarrow 0^{+}} t^{1-\beta} y_{i}(t)=y_{i}(1)
$$

Let $x=x_{1}-x_{2}, y=y_{1}-y_{2}$. Then $x, y$ satisfy the equations

$$
\begin{aligned}
& D_{0+}^{\alpha} x(t)=f\left(t, t^{1-\beta} y_{1}(t), D_{0+}^{\beta} y_{1}(t)\right)-f\left(t, t^{1-\beta} y_{2}(t), D_{0+}^{\beta} y_{2}(t)\right), \\
& D_{0+}^{\beta} y(t)=g\left(t, t^{1-\alpha} x_{1}(t), D_{0+}^{\alpha} x_{1}(t)\right)-g\left(t, t^{1-\alpha} x_{2}(t), D_{0_{+}}^{\alpha} x_{2}(t)\right) .
\end{aligned}
$$

Noting that $\operatorname{Im} L=\operatorname{Ker} Q$, we have

$$
\begin{aligned}
& \int_{0}^{1}(1-s)^{\alpha-1}\left[f\left(s, s^{1-\beta} y_{1}(s), D_{0+}^{\beta} y_{1}(s)\right)-f\left(s, s^{1-\beta} y_{2}(s), D_{0+}^{\beta} y_{2}(s)\right)\right] d s=0, \\
& \int_{0}^{1}(1-s)^{\beta-1}\left[g\left(s, s^{1-\alpha} x_{1}(s), D_{0+}^{\alpha} x_{1}(s)\right)-g\left(s, s^{1-\alpha} x_{2}(s), D_{0+}^{\alpha} x_{2}(s)\right)\right] d s=0,
\end{aligned}
$$

which imply there exist $t_{5}, t_{6} \in[0,1]$ such that

$$
\begin{aligned}
& f\left(t_{5}, t_{5}^{1-\beta} y_{1}\left(t_{5}\right), D_{0+}^{\beta} y_{1}\left(t_{5}\right)\right)-f\left(t_{5}, t_{5}^{1-\beta} y_{2}\left(t_{5}\right), D_{0+}^{\beta} y_{2}\left(t_{5}\right)\right)=0, \\
& g\left(t_{6}, t_{6}^{1-\alpha} x_{1}\left(t_{6}\right), D_{0+}^{\alpha} x_{1}\left(t_{6}\right)\right)-g\left(t_{6}, t_{6}^{1-\alpha} x_{2}\left(t_{6}\right), D_{0+}^{\alpha} x_{2}\left(t_{6}\right)\right)=0 .
\end{aligned}
$$


Basing on condition $\left(\mathrm{A}_{3}\right)$, we conclude that

$$
\left|t_{5}^{1-\beta} y\left(t_{5}\right)\right| \leq \frac{b}{a}\left\|D_{0+}^{\beta} y\right\|_{\infty}, \quad\left|t_{6}^{1-\alpha} x\left(t_{6}\right)\right| \leq \frac{d}{c}\left\|D_{0+}^{\alpha} x\right\|_{\infty} .
$$

Considering that

$$
I_{0+}^{\alpha} D_{0+}^{\alpha} x(t)=x(t)+c_{1} t^{\alpha-1}, \quad c_{1} \in \mathbb{R}, \quad I_{0+}^{\beta} D_{0+}^{\beta} y(t)=y(t)+c_{2} t^{\beta-1}, \quad c_{2} \in \mathbb{R}
$$

thus,

$$
c_{1}=\left.t^{1-\alpha} I_{0+}^{\alpha} D_{0+}^{\alpha} x(t)\right|_{t=t_{6}}-t_{6}^{1-\alpha} x\left(t_{6}\right), \quad c_{2}=\left.t^{1-\beta} I_{0+}^{\beta} D_{0+}^{\beta} y(t)\right|_{t=t_{5}}-t_{5}^{1-\beta} y\left(t_{5}\right) .
$$

Therefore, we can draw a fact

$$
\left|t^{1-\alpha} x(t)\right| \leq \kappa\left\|D_{0+}^{\alpha} x\right\|_{\infty}, \quad\left|t^{1-\beta} y(t)\right| \leq \mu\left\|D_{0+}^{\beta} y\right\|_{\infty} .
$$

On the other hand, using hypothesis $\left(\mathrm{A}_{2}\right)$ and (3.35)-(3.36), we find that

$$
\left|D_{0+}^{\alpha} x(t)\right| \leq p_{1}\left|t^{1-\beta} y(t)\right|+q_{1}\left|D_{0+}^{\beta} y(t)\right|, \quad\left|D_{0+}^{\beta} y(t)\right| \leq p_{2}\left|t^{1-\alpha} x(t)\right|+q_{2}\left|D_{0_{+}}^{\alpha} x(t)\right| .
$$

Consequently, we infer that

$$
\begin{aligned}
& \left|D_{0+}^{\alpha} x(t)\right| \leq p_{1}\left\|t^{1-\beta} y\right\|_{\infty}+q_{1}\left\|D_{0+}^{\beta} y\right\|_{\infty}, \\
& \left|D_{0_{+}}^{\beta} y(t)\right| \leq p_{2}\left\|t^{1-\alpha} x\right\|_{\infty}+q_{2}\left\|D_{0_{+}}^{\alpha} x\right\|_{\infty} .
\end{aligned}
$$

By Lemma 3.2, we obtain

$$
\begin{aligned}
\|(I-P)(x, y)\|_{X} & =\left\|K_{P} L(I-P)(x, y)\right\|_{X} \leq \Delta\|L(x, y)\|_{Z} \\
& =\Delta \max \left\{\left\|D_{0+}^{\alpha} x\right\|_{\infty},\left\|D_{0_{+}}^{\beta} y\right\|_{\infty}\right\} .
\end{aligned}
$$

From (3.38)-(3.40), one has

$$
\begin{aligned}
\|(x, y)\|_{X} \leq & \|P(x, y)\|_{X}+\|(I-P)(x, y)\|_{X} \\
\leq & \max \left\{\left\|t^{1-\beta} y\right\|_{\infty},\left\|t^{1-\alpha} x\right\|_{\infty}\right\}+\Delta \max \left\{\left\|D_{0+}^{\alpha} x\right\|_{\infty},\left\|D_{0_{+}}^{\beta} y\right\|_{\infty}\right\} \\
\leq & \max \left\{\mu\left\|D_{0_{+}}^{\beta} y\right\|_{\infty}, \kappa\left\|D_{0+}^{\alpha} x\right\|_{\infty}\right\}+\Delta \max \left\{\left\|D_{0+}^{\alpha} x\right\|_{\infty},\left\|D_{0_{+}}^{\beta} y\right\|_{\infty}\right\} \\
= & \max \left\{\kappa\left\|D_{0+}^{\alpha} x\right\|_{\infty}+\Delta\left\|D_{0+}^{\beta} y\right\|_{\infty},(\kappa+\Delta)\left\|D_{0+}^{\alpha} x\right\|_{\infty},\right. \\
& \left.\mu\left\|D_{0+}^{\beta} y\right\|_{\infty}+\Delta\left\|D_{0+}^{\alpha} x\right\|_{\infty},(\mu+\Delta)\left\|D_{0+}^{\beta} y\right\|_{\infty}\right\} .
\end{aligned}
$$

Proceeding as in the proof of Lemma 3.4, we divide the proof in four cases.

Case 1. $\|(x, y)\|_{X} \leq \kappa\left\|D_{0+}^{\alpha} x\right\|_{\infty}+\Delta\left\|D_{0+}^{\beta} y\right\|_{\infty}$. By (3.20) and (3.39), we get

$$
\begin{aligned}
\|(x, y)\|_{X} & \leq \kappa\left\|D_{0+}^{\alpha} x\right\|_{\infty}+\Delta\left\|D_{0_{+}}^{\beta} y\right\|_{\infty} \\
& \leq \kappa\left(p_{1}\left\|t^{1-\beta} y\right\|_{\infty}+q_{1}\left\|D_{0_{+}}^{\beta} y\right\|_{\infty}\right)+\Delta\left(p_{2}\left\|t^{1-\alpha} x\right\|_{\infty}+q_{2}\left\|D_{0_{+}}^{\alpha} x\right\|_{\infty}\right)
\end{aligned}
$$




$$
\leq \kappa\left(p_{1}\left\|t^{1-\beta} y\right\|_{\infty}+q_{1}\|(x, y)\|_{X}\right)+\Delta\left(p_{2}+q_{2}\right)\|(x, y)\|_{X},
$$

and

$$
\begin{aligned}
\|(x, y)\|_{X} & \leq \kappa\left\|D_{0+}^{\alpha} x\right\|_{\infty}+\Delta\left\|D_{0+}^{\beta} y\right\|_{\infty} \\
& \leq \kappa\left(p_{1}\left\|t^{1-\beta} y\right\|_{\infty}+q_{1}\left\|D_{0+}^{\beta} y\right\|_{\infty}\right)+\Delta\left(p_{2}\left\|t^{1-\alpha} x\right\|_{\infty}+q_{2}\left\|D_{0+}^{\alpha} x\right\|_{\infty}\right) \\
& \leq \kappa\left(p_{1}+q_{1}\right)\|(x, y)\|_{X}+\Delta p_{2}\left\|t^{1-\alpha} x\right\|_{\infty}+\Delta q_{2}\|(x, y)\|_{X} .
\end{aligned}
$$

Again, by (3.20), we obtain

$$
\begin{aligned}
\left\|t^{1-\beta} y\right\|_{\infty} & \leq \frac{\kappa p_{1}}{1-\left[\kappa q_{1}+\Delta\left(p_{2}+q_{2}\right)\right]}\left\|t^{1-\beta} y\right\|_{\infty}, \\
\left\|t^{1-\alpha} x\right\|_{\infty} & \leq \frac{\Delta p_{2}}{1-\left[\kappa\left(p_{1}+q_{1}\right)+\Delta q_{2}\right]}\left\|t^{1-\alpha} x\right\|_{\infty} .
\end{aligned}
$$

In view of condition (3.34), we have

$$
\left\|t^{1-\alpha} x\right\|_{\infty}=0, \quad\left\|t^{1-\beta} y\right\|_{\infty}=0
$$

As a result, we get $x_{1}=x_{2}, y_{1}=y_{2}$.

Case 2. $\|(x, y)\|_{X} \leq(\kappa+\Delta)\left\|D_{0_{+}}^{\alpha} x\right\|_{\infty}$. Then (3.39) and (3.20) imply

$$
\begin{aligned}
\|(x, y)\|_{X} & \leq(\kappa+\Delta)\left\|D_{0+}^{\alpha} x\right\|_{\infty} \\
& \leq(\kappa+\Delta)\left[p_{1}\left\|t^{1-\beta} y\right\|_{\infty}+q_{1}\left\|D_{0+}^{\beta} y\right\|_{\infty}\right] \\
& \leq(\kappa+\Delta) p_{1}\left\|t^{1-\beta} y\right\|_{\infty}+q_{1}(\kappa+\Delta)\|(x, y)\|_{X},
\end{aligned}
$$

and

$$
\begin{aligned}
\|(x, y)\|_{X} & \leq(\kappa+\Delta)\left\|D_{0+}^{\alpha} x\right\|_{\infty} \\
& \leq(\kappa+\Delta)\left[p_{1}\left\|t^{1-\beta} y\right\|_{\infty}+q_{1} p_{2}\left\|t^{1-\alpha} x\right\|_{\infty}+q_{1} q_{2}\left\|D_{0+}^{\alpha} x\right\|_{\infty}\right] \\
& \leq(\kappa+\Delta) q_{1} p_{2}\left\|t^{1-\alpha} x\right\|_{\infty}+(\kappa+\Delta)\left(p_{1}+q_{1} q_{2}\right)\|(x, y)\|_{X} .
\end{aligned}
$$

Using (3.20), we derive

$$
\begin{aligned}
\left\|t^{1-\beta} y\right\|_{\infty} & \leq \frac{(\kappa+\Delta) p_{1}}{1-(\kappa+\Delta) q_{1}}\left\|t^{1-\beta} y\right\|_{\infty}, \\
\left\|t^{1-\alpha} x\right\|_{\infty} & \leq \frac{(\kappa+\Delta) q_{1} p_{2}}{1-(\kappa+\Delta)\left(p_{1}+q_{1} q_{2}\right)}\left\|t^{1-\alpha} x\right\|_{\infty} .
\end{aligned}
$$

According to assumption (3.34), we obtain

$$
\left\|t^{1-\alpha} x\right\|_{\infty}=0, \quad\left\|t^{1-\beta} y\right\|_{\infty}=0 .
$$

Consequently, $x_{1}=x_{2}, y_{1}=y_{2}$. 
Case 3. $\|(x, y)\|_{X} \leq \mu\left\|D_{0+}^{\beta} y\right\|_{\infty}+\Delta\left\|D_{0_{+}}^{\alpha} x\right\|_{\infty}$. By a method similar to that used in Case 1, we can conclude that

$$
\begin{aligned}
\left\|t^{1-\beta} y\right\|_{\infty} & \leq \frac{\Delta p_{1}}{1-\left[\Delta q_{1}+\mu\left(p_{2}+q_{2}\right)\right]}\left\|t^{1-\beta} y\right\|_{\infty}, \\
\left\|t^{1-\alpha} x\right\|_{\infty} & \leq \frac{\mu p_{2}}{1-\left[\Delta\left(p_{1}+q_{1}\right)+\mu q_{2}\right]}\left\|t^{1-\alpha} x\right\|_{\infty} .
\end{aligned}
$$

Case 4. $\|(x, y)\|_{X} \leq(\mu+\Delta)\left\|D_{0+}^{\beta} y\right\|_{\infty}$. Similar to the analysis in Case 2, we can deduce that

$$
\begin{aligned}
\left\|t^{1-\beta} y\right\|_{\infty} & \leq \frac{(\mu+\Delta) p_{1} q_{2}}{1-(\mu+\Delta)\left(p_{2}+q_{1} q_{2}\right)}\left\|t^{1-\beta} y\right\|_{\infty} \\
\left\|t^{1-\alpha} x\right\|_{\infty} & \leq \frac{(\mu+\Delta) p_{2}}{1-(\mu+\Delta) q_{2}}\left\|t^{1-\alpha} x\right\|_{\infty} .
\end{aligned}
$$

From (3.34), (3.42), and (3.43), we also obtain that

$$
\left\|t^{1-\alpha} x\right\|_{\infty}=0, \quad\left\|t^{1-\beta} y\right\|_{\infty}=0
$$

that is, $x_{1}=x_{2}, y_{1}=y_{2}$. In summary, BVP (1.1) has a unique continuous solution in $X$.

\section{Example}

Example 4.1 Consider the boundary value problem

$$
\left\{\begin{array}{l}
D_{0+}^{1 / 2} x(t)=f\left(t, t^{1 / 2} y(t), D_{0+}^{1 / 2} y(t)\right), \quad t \in[0,1], \\
D_{0+}^{1 / 2} y(t)=g\left(t, t^{1 / 2} x(t), D_{0+}^{1 / 2} x(t)\right), \\
\lim _{t \rightarrow 0^{+}} t^{1 / 2} x(t)=x(1), \quad \lim _{t \rightarrow 0^{+}} t^{1 / 2} y(t)=y(1) .
\end{array}\right.
$$

Corresponding to problem (1.1), here

$$
\begin{aligned}
& \alpha=\beta=\frac{1}{2}, \\
& f\left(t, t^{1 / 2} y(t), D_{0+}^{1 / 2} y(t)\right)=\left\{\begin{array}{l}
\frac{11}{50} t^{2} \sin \left|t^{1 / 2} y(t)\right|+\frac{t}{2} \sin D_{0+}^{1 / 2} y(t)+\frac{4}{5}, \quad\left|t^{1 / 2} y(t)\right| \leq 3, \\
\frac{11}{50} t^{2} \sin 3+\frac{t}{2} \sin D_{0+}^{1 / 2} y(t)+\frac{4}{5}, \quad\left|t^{1 / 2} y(t)\right| \geq 3,
\end{array}\right. \\
& g\left(t, t^{1 / 2} x(t), D_{0+}^{1 / 2} x(t)\right)= \begin{cases}\frac{1}{5} t^{2}\left|t^{1 / 2} x(t)\right|+\frac{t}{2} \sin D_{0+}^{1 / 2} x(t)+\frac{2}{3}, & \left|t^{1 / 2} y(t)\right| \leq 1, \\
\frac{1}{5} t^{2}+\frac{t}{2} \sin D_{0+}^{1 / 2} x(t)+\frac{2}{3}, & \left|t^{1 / 2} y(t)\right| \geq 1 .\end{cases}
\end{aligned}
$$

Let

$$
\begin{array}{lll}
\gamma_{1}(t)=\frac{11}{50} t^{2}, & \eta_{1}(t)=\frac{t}{2}, & \omega_{1}(t)=\frac{4}{5}, \\
\gamma_{2}(t)=\frac{1}{5} t^{2}, & \eta_{2}(t)=\frac{t}{2}, & \omega_{2}(t)=\frac{2}{3} .
\end{array}
$$

Then $\left(\mathrm{A}_{1}\right)$ holds and

$$
\gamma_{1}=\frac{11}{50}, \quad \eta_{1}=\eta_{2}=\frac{1}{2}, \quad \omega_{1}=\frac{4}{5}, \quad \gamma_{2}=\frac{1}{5}, \quad \omega_{2}=\frac{2}{3},
$$




$$
\begin{aligned}
& \sigma_{2}=\frac{\gamma_{2} \eta_{1}}{1-\eta_{1} \eta_{2}}=\frac{2}{15}, \quad \rho_{3}=\frac{\gamma_{1} \eta_{2}}{1-\eta_{1} \eta_{2}}=\frac{11}{75}, \quad \rho_{2}=\frac{\gamma_{2}}{1-\eta_{1} \eta_{2}}=\frac{4}{15}, \\
& \sigma_{3}=\frac{\gamma_{1}}{1-\eta_{1} \eta_{2}}=\frac{22}{75} .
\end{aligned}
$$

Accordingly,

$$
\begin{aligned}
& \eta_{1} \eta_{2}=\frac{1}{4}<1, \quad \Gamma(\alpha+1)=\frac{\sqrt{\pi}}{2}>2 \sigma_{2}=\frac{4}{15}, \quad \Gamma(\beta+1)=\frac{\sqrt{\pi}}{2}>2 \rho_{3}=\frac{22}{75}, \\
& \left(\Gamma(\alpha+1)-2 \sigma_{2}\right)\left(\Gamma(\beta+1)-2 \rho_{3}\right)=\left(\frac{\sqrt{\pi}}{2}-\frac{4}{15}\right)\left(\frac{\sqrt{\pi}}{2}-\frac{22}{75}\right)>\frac{352}{1125}=4 \rho_{2} \sigma_{3} .
\end{aligned}
$$

Consequently, (3.8) holds. Since

$$
\begin{aligned}
& N_{1} y=\left\{\begin{array}{l}
\frac{11}{50} t^{2} \sin \left|t^{1 / 2} y(t)\right|+\frac{t}{2} \sin D_{0+}^{1 / 2} y(t)+\frac{4}{5}, \quad\left|t^{1 / 2} y(t)\right| \leq 3, \\
\frac{11}{50} t^{2} \sin 3+\frac{t}{2} \sin D_{0+}^{1 / 2} y(t)+\frac{4}{5}, \quad\left|t^{1 / 2} y(t)\right| \geq 3,
\end{array}\right. \\
& N_{2} x=\left\{\begin{array}{l}
\frac{1}{5} t^{2}\left|t^{1 / 2} x(t)\right|+\frac{t}{2} \sin D_{0+}^{1 / 2} x(t)+\frac{2}{3}, \quad\left|t^{1 / 2} y(t)\right| \leq 1, \\
\frac{1}{5} t^{2}+\frac{t}{2} \sin D_{0+}^{1 / 2} x(t)+\frac{2}{3}, \quad\left|t^{1 / 2} y(t)\right| \geq 1,
\end{array}\right.
\end{aligned}
$$

and

$$
\begin{aligned}
& N_{1}\left(c_{2} t^{\beta-1}\right)=N_{1}\left(c_{2} t^{-1 / 2}\right)=\left\{\begin{array}{l}
\frac{11}{50} t^{2} \sin \left|c_{2}\right|+\frac{4}{5}, \quad\left|c_{2}\right| \leq 3, \\
\frac{11}{50} t^{2} \sin 3+\frac{4}{5}, \quad\left|c_{2}\right| \geq 3,
\end{array}\right. \\
& N_{2}\left(c_{1} t^{\alpha-1}\right)=N_{1}\left(c_{1} t^{-1 / 2}\right)=\left\{\begin{array}{l}
\frac{1}{5} t^{2}\left|c_{1}\right|+\frac{2}{3}, \quad\left|c_{1}\right| \leq 1, \\
\frac{1}{5} t^{2}+\frac{2}{3}, \quad\left|c_{1}\right| \geq 1 .
\end{array}\right.
\end{aligned}
$$

So if we put $B_{1}=G_{1}=1, B_{2}=G_{2}=3$, then we have

$$
\begin{aligned}
& N_{1} y=\frac{11}{50} t^{2} \sin 3+\frac{t}{2} \sin D_{0+}^{1 / 2} y(t)+\frac{4}{5} \geq \frac{2}{25}>0, \quad\left|t^{1 / 2} y(t)\right| \geq 3, \\
& N_{1}\left(c_{2} t^{\beta-1}\right)=N_{1}\left(c_{2} t^{-1 / 2}\right)=\frac{11}{50} t^{2} \sin 3+\frac{4}{5} \geq \frac{29}{50}>0, \quad\left|c_{2}\right| \geq 3, \\
& N_{2} x=\frac{1}{5} t^{2}+\frac{t}{2} \sin D_{0+}^{1 / 2} x(t)+\frac{2}{3} \geq \frac{1}{6}>0, \quad\left|t^{1 / 2} y(t)\right| \geq 1, \\
& N_{2}\left(c_{1} t^{\alpha-1}\right)=N_{1}\left(c_{1} t^{-1 / 2}\right)=\frac{1}{5} t^{2}+\frac{2}{3} \geq \frac{2}{3}>0, \quad\left|c_{1}\right| \geq 1 .
\end{aligned}
$$

Therefore, $\left(\mathrm{A}_{4}\right)$ and $\left(\mathrm{A}_{5}\right)$ hold. By Theorem 3.1, we can conclude that BVP (4.1) has at least one solution.

Remark 4.1 Obviously, for BVP (4.1), condition $\left(\mathrm{A}_{2}\right)$ is not valid and (3.9) does not hold.

In fact, we can obtain that

$$
\frac{2}{\Gamma(\alpha+1)}\left(\gamma_{1}+\eta_{1}\right)+\Delta\left(\gamma_{2}+\eta_{2}\right)=\frac{4}{\sqrt{\pi}} \cdot \frac{18}{25}+\frac{\pi+2 \sqrt{\pi}}{\pi} \cdot \frac{7}{10}>1 .
$$

So that (3.9) is not true. 
Example 4.2 Consider the boundary value problem

$$
\left\{\begin{array}{l}
D_{0+}^{1 / 2} x(t)=\frac{t^{2}}{5} \sin t^{1 / 2} y(t)+\frac{4 t}{15} \sin D_{0^{+}}^{1 / 2} y(t)+\frac{1}{2}, \quad t \in[0,1] \\
D_{0^{1}}^{1 / 2} y(t)=\frac{t^{2}}{7} \sin t^{1 / 2} x(t)+\frac{2 t}{7} \sin D_{0^{1}}^{1 / 2} x(t)+\frac{1}{2}, \\
\lim _{t \rightarrow 0^{+}} t^{1 / 2} x(t)=x(1), \quad \lim _{t \rightarrow 0^{+}} t^{1 / 2} y(t)=y(1) .
\end{array}\right.
$$

Corresponding to problem (1.1), here

$$
\begin{aligned}
& \alpha=\beta=\frac{1}{2}, \\
& f\left(t, t^{1-\beta} y(t), D_{0+}^{\beta} y(t)\right)=\frac{t^{2}}{5} \sin t^{1 / 2} y(t)+\frac{4 t}{15} \sin D_{0+}^{1 / 2} y(t)+\frac{1}{2}, \\
& g\left(t, t^{1-\alpha} x(t), D_{0+}^{\alpha} x(t)\right)=\frac{t^{2}}{7} \sin t^{1 / 2} x(t)+\frac{2 t}{7} \sin D_{0+}^{1 / 2} x(t)+\frac{1}{2} .
\end{aligned}
$$

Let

$$
p_{1}(t)=\frac{t^{2}}{5}, \quad q_{1}(t)=\frac{4 t}{15}, \quad p_{2}(t)=\frac{t^{2}}{7}, \quad q_{2}(t)=\frac{2 t}{7},
$$

then $p_{1}=\frac{1}{5}, q_{1}=\frac{4}{15}, p_{2}=\frac{1}{7}, q_{2}=\frac{2}{7}$. We can easily check that $\left(\mathrm{A}_{2}\right)$ holds and

$$
\begin{aligned}
& \Gamma(\alpha+1) \Gamma(\beta+1) \\
& \quad=\frac{\pi}{4}>\frac{(2+\sqrt{\pi})^{2}}{20}=(1+\Gamma(\alpha+1))(1+\Gamma(\beta+1))\left(p_{1}+q_{1}\right)\left(p_{2}+q_{2}\right) .
\end{aligned}
$$

By Theorem 3.2, BVP (4.2) has at least one solution. If we let

$$
\begin{array}{lll}
\gamma_{1}(t)=\frac{t^{2}}{5}, & \eta_{1}(t)=\frac{4 t}{15}, & \omega_{1}(t)=\frac{1}{2}, \\
\gamma_{2}(t)=\frac{t^{2}}{7}, & \eta_{2}(t)=\frac{2 t}{7}, & \omega_{2}(t)=\frac{1}{2},
\end{array}
$$

then $\left(\mathrm{A}_{1}\right)$ holds and

$$
\begin{aligned}
& \gamma_{1}=\frac{1}{5}, \quad \eta_{1}=\frac{4}{15}, \quad \omega_{1}=\omega_{2}=\frac{1}{2}, \quad \gamma_{2}=\frac{1}{7}, \quad \eta_{2}=\frac{2}{7}, \\
& \sigma_{2}=\frac{\gamma_{2} \eta_{1}}{1-\eta_{1} \eta_{2}}=\frac{4}{97}, \quad \rho_{3}=\frac{\gamma_{1} \eta_{2}}{1-\eta_{1} \eta_{2}}=\frac{6}{97}, \quad \rho_{2}=\frac{\gamma_{2}}{1-\eta_{1} \eta_{2}}=\frac{15}{97}, \\
& \sigma_{3}=\frac{\gamma_{1}}{1-\eta_{1} \eta_{2}}=\frac{21}{97} .
\end{aligned}
$$

Therefore,

$$
\begin{aligned}
& \eta_{1} \eta_{2}=\frac{8}{105}<1, \quad \Gamma(\alpha+1)=\frac{\sqrt{\pi}}{2}>2 \sigma_{2}=\frac{8}{97}, \quad \Gamma(\beta+1)=\frac{\sqrt{\pi}}{2}>2 \rho_{3}=\frac{12}{97}, \\
& \left(\Gamma(\alpha+1)-2 \sigma_{2}\right)\left(\Gamma(\beta+1)-2 \rho_{3}\right)=\left(\frac{\sqrt{\pi}}{2}-\frac{8}{97}\right)\left(\frac{\sqrt{\pi}}{2}-\frac{12}{97}\right)>\frac{1260}{9409}=4 \rho_{2} \sigma_{3},
\end{aligned}
$$


that is, (3.8) holds. Since

$$
\begin{aligned}
& N_{1} y=\frac{t^{2}}{5} \sin t^{1 / 2} y(t)+\frac{4 t}{15} \sin D_{0+}^{1 / 2} y(t)+\frac{1}{2} \geq \frac{1}{30}>0 \\
& N_{1}\left(c_{2} t^{\beta-1}\right)=N_{1}\left(c_{2} t^{-1 / 2}\right)=\frac{t^{2}}{5} \sin c_{2}+\frac{1}{2} \geq \frac{3}{10}>0, \\
& N_{2} x=\frac{t^{2}}{7} \sin t^{1 / 2} x(t)+\frac{2 t}{7} \sin D_{0+}^{1 / 2} x(t)+\frac{1}{2} \geq \frac{1}{14}>0, \\
& N_{2}\left(c_{1} t^{\alpha-1}\right)=N_{1}\left(c_{1} t^{-1 / 2}\right)=\frac{t^{2}}{7} \sin c_{1}+\frac{1}{2} \geq \frac{4}{15}>0 .
\end{aligned}
$$

Then, for any $B_{i}>0$ and $G_{i}>0,(i=1,2)$, we have $\left(\mathrm{A}_{4}\right)$ and $\left(\mathrm{A}_{5}\right)$ hold. By Theorem 3.1 , we can also obtain that BVP (4.2) has at least one solution.

Remark 4.2 The existence result of BVP (4.2) cannot be obtained by verifying conditions $\left(A_{1}\right),\left(A_{4}\right),\left(A_{5}\right)$, and (3.9) of Theorem 3.1.

In fact, we can check that

$$
\frac{2}{\Gamma(\alpha+1)}\left(\gamma_{1}+\eta_{1}\right)+\Delta\left(\gamma_{2}+\eta_{2}\right)=\frac{4}{\sqrt{\pi}} \cdot \frac{7}{15}+\frac{\sqrt{\pi}+2}{\sqrt{\pi}} \cdot \frac{3}{7}=\frac{286+45 \sqrt{\pi}}{105 \sqrt{\pi}}>1 .
$$

This implies that (3.9) does not hold.

Example 4.3 Consider the following fractional boundary value problem:

$$
\left\{\begin{array}{l}
D_{0+}^{1 / 2} x(t)=\frac{t^{2}+3}{30} \sin t^{1 / 2} y(t)+\frac{t}{20} \sin D_{0+}^{1 / 2} y(t)+\frac{1}{4}, \quad t \in[0,1] \\
D_{0+}^{1 / 2} y(t)=\frac{t^{2}+1}{14} \sin t^{1 / 2} x(t)+\frac{t}{28} \sin D_{0+}^{1 / 2} x(t)+\frac{1}{5} \\
\lim _{t \rightarrow 0^{+}} t^{1 / 2} x(t)=x(1), \quad \lim _{t \rightarrow 0^{+}} t^{1 / 2} y(t)=y(1) .
\end{array}\right.
$$

Corresponding to problem (1.1), here

$$
\begin{aligned}
& \alpha=\beta=\frac{1}{2}, \\
& f\left(t, t^{1-\beta} y(t), D_{0+}^{\beta} y(t)\right)=\frac{t^{2}+3}{30} \sin t^{1 / 2} y(t)+\frac{t}{20} \sin D_{0+}^{1 / 2} y(t)+\frac{1}{4}, \\
& g\left(t, t^{1-\alpha} x(t), D_{0+}^{\alpha} x(t)\right)=\frac{t^{2}+1}{14} \sin t^{1 / 2} x(t)+\frac{t}{28} \sin D_{0+}^{1 / 2} x(t)+\frac{1}{5} .
\end{aligned}
$$

Let

$$
p_{1}(t)=\frac{1}{30} t^{2}+\frac{1}{10}, \quad q_{1}(t)=\frac{1}{20} t, \quad p_{2}(t)=\frac{1}{14} t^{2}+\frac{1}{14}, \quad q_{2}(t)=\frac{1}{28} t .
$$

Then

$$
\begin{aligned}
& p_{1}=\frac{2}{15}, \quad q_{1}=\frac{1}{20}, \quad p_{2}=\frac{1}{7}, \quad q_{2}=\frac{1}{28}, \quad \Delta=\frac{\pi+2 \sqrt{\pi}}{\pi}, \\
& p_{1}+q_{1}=\frac{11}{60}>\frac{5}{28}=p_{2}+q_{2} .
\end{aligned}
$$


Choose $a=\frac{1}{10}, b=\frac{1}{20}, c=\frac{1}{14}, d=\frac{1}{28}$. It is easy to show that $\left(\mathrm{A}_{2}\right)$ and $\left(\mathrm{A}_{3}\right)$ hold. Since

$$
\begin{aligned}
\Gamma(\alpha+1) \Gamma(\beta+1) & =\frac{\pi}{4}>\frac{11}{1344}(2+\sqrt{\pi})^{2} \\
& =(1+\Gamma(\alpha+1))(1+\Gamma(\beta+1))\left(p_{1}+q_{1}\right)\left(p_{2}+q_{2}\right) .
\end{aligned}
$$

By Theorem 3.2, BVP (4.3) has at least one solution. We also can check that

$$
\begin{aligned}
&(\kappa+\Delta)\left(p_{1}+q_{1}\right)=\left(\frac{2}{\Gamma(3 / 2)}+\frac{1}{2}+\frac{\pi+2 \sqrt{\pi}}{\pi}\right)\left(\frac{2}{15}+\frac{1}{20}\right) \\
&=\frac{11 \sqrt{\pi}+44}{40 \sqrt{\pi}}:=\Xi<1, \\
& \kappa\left(p_{1}+q_{1}\right)+\Delta\left(p_{2}+q_{2}\right)<\Xi<1, \\
& \mu\left(p_{2}+q_{2}\right)+\Delta\left(p_{1}+q_{1}\right)=\kappa\left(p_{2}+q_{2}\right)+\Delta\left(p_{1}+q_{1}\right)<\Xi<1, \\
&(\mu+\Delta)\left(p_{2}+q_{2}\right)=(\kappa+\Delta)\left(p_{2}+q_{2}\right)<\Xi<1 .
\end{aligned}
$$

By Theorem 3.3, BVP (4.3) has a unique solution. If we let

$$
\begin{array}{lll}
\gamma_{1}(t)=\frac{t^{2}+3}{30}, & \eta_{1}(t)=\frac{t}{20}, & \omega_{1}(t)=\frac{1}{4}, \\
\gamma_{2}(t)=\frac{t^{2}+1}{14}, & \eta_{2}(t)=\frac{t}{28}, & \omega_{2}(t)=\frac{1}{5},
\end{array}
$$

then $\left(\mathrm{A}_{1}\right)$ holds and

$$
\begin{aligned}
& \gamma_{1}=\frac{2}{15}, \quad \eta_{1}=\frac{1}{20}, \quad \omega_{1}=\frac{1}{4}, \quad \gamma_{2}=\frac{1}{7}, \quad \eta_{2}=\frac{1}{28}, \quad \omega_{2}=\frac{1}{5}, \\
& \gamma_{2}+\eta_{2}=\frac{5}{28}<\frac{11}{60}=\gamma_{1}+\eta_{1}, \\
& \sigma_{2}=\frac{\gamma_{2} \eta_{1}}{1-\eta_{1} \eta_{2}}=\frac{4}{559}, \quad \rho_{3}=\frac{\gamma_{1} \eta_{2}}{1-\eta_{1} \eta_{2}}=\frac{8}{1677}, \quad \rho_{2}=\frac{\gamma_{2}}{1-\eta_{1} \eta_{2}}=\frac{80}{559}, \\
& \sigma_{3}=\frac{\gamma_{1}}{1-\eta_{1} \eta_{2}}=\frac{224}{1677} .
\end{aligned}
$$

Thus,

$$
\begin{aligned}
& \eta_{1} \eta_{2}=\frac{1}{560}<1, \quad \Gamma(\alpha+1)=\frac{\sqrt{\pi}}{2}>2 \sigma_{2}=\frac{8}{559}, \quad \Gamma(\beta+1)=\frac{\sqrt{\pi}}{2}>2 \rho_{3}=\frac{16}{1677}, \\
& \left(\Gamma(\alpha+1)-2 \sigma_{2}\right)\left(\Gamma(\beta+1)-2 \rho_{3}\right) \\
& \quad=\left(\frac{\sqrt{\pi}}{2}-\frac{8}{559}\right)\left(\frac{\sqrt{\pi}}{2}-\frac{16}{1677}\right)>\frac{71,680}{937,443}=4 \rho_{2} \sigma_{3},
\end{aligned}
$$

and

$$
\begin{aligned}
\frac{2}{\Gamma(\alpha+1)}\left(\gamma_{1}+\eta_{1}\right)+\Delta\left(\gamma_{2}+\eta_{2}\right) & \leq\left(\frac{2}{\Gamma(\alpha+1)}+\Delta\right)\left(\gamma_{1}+\eta_{1}\right) \\
& =\left(\frac{2}{\Gamma(3 / 2)}+\frac{\pi+2 \sqrt{\pi}}{\pi}\right)\left(\frac{2}{15}+\frac{1}{20}\right)<\Xi<1,
\end{aligned}
$$




$$
\begin{aligned}
& \frac{2}{\Gamma(\beta+1)}\left(\gamma_{2}+\eta_{2}\right)+\Delta\left(\gamma_{1}+\eta_{1}\right) \leq \frac{2}{\Gamma(\alpha+1)}\left(\gamma_{1}+\eta_{1}\right)+\Delta\left(\gamma_{2}+\eta_{2}\right)<1, \\
& \left(\frac{2}{\Gamma(\beta+1)}+\Delta\right)\left(\gamma_{2}+\eta_{2}\right) \leq\left(\frac{2}{\Gamma(\alpha+1)}+\Delta\right)\left(\gamma_{1}+\eta_{1}\right)<1 .
\end{aligned}
$$

That is, both (3.8) and (3.9) hold because

$$
\begin{aligned}
& N_{1} y=\frac{t^{2}+3}{30} \sin t^{1 / 2} y(t)+\frac{t}{20} \sin D_{0+}^{1 / 2} y(t)+\frac{1}{4} \geq \frac{1}{15}>0, \\
& N_{1}\left(c_{2} t^{\beta-1}\right)=N_{1}\left(c_{2} t^{-1 / 2}\right)=\frac{t^{2}+3}{30} \sin c_{2}+\frac{1}{4} \geq \frac{7}{60}>0, \\
& N_{2} x=\frac{t^{2}+1}{14} \sin t^{1 / 2} x(t)+\frac{t}{28} \sin D_{0+}^{1 / 2} x(t)+\frac{1}{5} \geq \frac{3}{140}>0, \\
& N_{2}\left(c_{1} t^{\alpha-1}\right)=N_{1}\left(c_{1} t^{-1 / 2}\right)=\frac{t^{2}+1}{14} \sin c_{1}+\frac{1}{5} \geq \frac{2}{35}>0 .
\end{aligned}
$$

Therefore, for any $B_{i}>0$ and $G_{i}>0,(i=1,2)\left(\mathrm{A}_{4}\right)$ and $\left(\mathrm{A}_{5}\right)$ hold, which means the existence result of BVP (4.3) can be obtained by Theorem 3.1.

\section{Conclusion}

In the present paper, we investigate the existence and uniqueness of solutions for the coupled systems of nonlinear implicit fractional periodic boundary value problems in the frame of Riemann-Liouville fractional derivative. By using Theorems 2.1 and 2.2, the new existence and uniqueness results are established. The results in papers $[29,30]$ are improved and extended in this paper. First, we extend the results of $[29,30]$ to coupled systems; second, in [29,30], the authors only studied the existence results based on Lemma 2.1 and established existence theorems under condition $\left(\mathrm{A}_{2}\right)$. Our results show that the existence results can also be obtained under condition $\left(\mathrm{A}_{1}\right)$. Besides, compared with [40-43], we used a different technique to prove that $\Omega_{1}$ is bounded (see Lemma 3.4, the first way). By Remark 3.1, we show that the first way is superior to the second way used by [40-43]. Finally, our main results are well illustrated with the aid of several interesting examples.

\section{Acknowledgements}

The authors wish to express their sincere appreciation to the editor and the anonymous referees for their valuable comments and suggestions.

\section{Funding}

This research is supported by the Fundamental Research Funds for the Central Universities (2017XKQY090).

Availability of data and materials

Data sharing not applicable to this article as no datasets were generated or analysed during the current study.

Competing interests

The authors declare that they have no competing interests.

Authors' contributions

The authors have made equal contributions to each part of this paper. All the authors read and approved the final manuscript.

\section{Publisher's Note}

Springer Nature remains neutral with regard to jurisdictional claims in published maps and institutional affiliations. 


\section{References}

1. Samko, S.G., Kilbas, A.A., Marichev, O.I.: Fractional Integrals and Derivatives: Theory and Applications. Gordon \& Breach, Switzerland (1993)

2. Kilbas, A.A., Srivastava, H.M., Trujillo, J.J.: Theory and Applications of Fractional Differential Equations. North-Holland Mathematics Studies. Elsevier, Amsterdam (2006)

3. Baleanu, D., Guvenc, Z.B., Machado, J.A.T.: New Trends in Nanotechnology and Fractional Calculus Applications. Springer, Netherlands (2010)

4. Sheng, H., Chen, Y.Q., Qiu, T.S.: Fractional Processes and Fractional-Order Signal Processing: Techniques and Applications. Springer, New York (2012)

5. Kaczorek, T., Rogowski, K.: Fractional Linear Systems and Electrical Circuits. Springer, Berlin (2015)

6. Tepljakov, A.: Fractional-Order Modeling and Control of Dynamic Systems. Springer, Berlin (2017)

7. Vyawahare, V., Nataraj, P.S.V.: Fractional-Order Modeling of Nuclear Reactor: From Subdiffusive Neutron Transport to Control-Oriented Models: A Systematic Approach. Springer, Berlin (2018)

8. Laskin, N.: Fractional market dynamics. Physica A 287, 482-492 (2000)

9. Agarwal, R., Hristova, S., O'Regan, D.: Iterative techniques for the initial value problem for Caputo fractional differential equations with non-instantaneous impulses. Appl. Math. Comput. 334, 407-421 (2018)

10. Ahmad, B., Luca, R.: Existence of solutions for a system of fractional differential equations with coupled nonloca boundary conditions. Fract. Calc. Appl. Anal. 21, 423-441 (2018)

11. Alsaedi, A., Aljoudi, S., Ahmad, B.: Existence of solutions for Riemann-Liouville type coupled systems of fractional integro-differential equations and boundary conditions. Electron. J. Differ. Equ. 2016, 211 (2016)

12. Bai, Z.B., Chen, Y.Q., Lian, H.R., Sun, S.J.: On the existence of blow up solutions for a class of fractional differential equations. Fract. Calc. Appl. Anal. 17, 1175-1187 (2014)

13. Bai, Z.B., Zhang, Y.H.: Solvability of fractional three-point boundary value problems with nonlinear growth. Appl. Math. Comput. 218, 1719-1725 (2011)

14. Henderson, J., Luca, R.: Systems of Riemann-Liouville fractional equations with multi-point boundary conditions. Appl. Math. Comput. 309, 303-323 (2017)

15. Ma, T.F., Yan, B.Q.: The multiplicity solutions for nonlinear fractional differential equations of Riemann-Liouville type. Fract. Calc. Appl. Anal. 21, 801-818 (2018)

16. Padhi, S., Graef, J.R., Pati, S.: Multiple positive solutions for a boundary value problem with nonlinear nonlocal Riemann-Stieltjes integral boundary conditions. Fract. Calc. Appl. Anal. 21, 716-745 (2018)

17. Song, Q.L., Bai, Z.B.: Positive solutions of fractional differential equations involving the Riemann-Stieltjes integral boundary condition. Adv. Differ. Equ. 2018, 183 (2018)

18. Song, Q.L., Dong, X.Y., Bai, Z.B., Chen, B.: Existence for fractional Dirichlet boundary value problem under barrier strip conditions. J. Nonlinear Sci. Appl. 10, 3592-3598 (2017)

19. Zhang, X.Q., Zhong, Q.Y.: Uniqueness of solution for higher-order fractional differential equations with conjugate type integral conditions. Fract. Calc. Appl. Anal. 20, 1471-1484 (2017)

20. Henderson, J., Luca, R.: Boundary Value Problems for Systems of Differential, Difference and Fractional Equations. Elsevier, Amsterdam (2016)

21. Ahmad, B., Alsaedi, A., Ntouyas, S.K., Tariboon, J.: Hadamard-Type Fractional Differential Equations, Inclusions and Inequalities. Springer, Berlin (2017)

22. Cabada, A., Kisela, T.: Existence of positive periodic solutions of some nonlinear fractional differential equations. Commun. Nonlinear Sci. Numer. Simul. 50, 51-67 (2017)

23. Staněk, S.: Periodic problem for two-term fractional differential equations. Fract. Calc. Appl. Anal. 20, 662-678 (2017)

24. Ahmad, B., Nieto, J.J., Alsaedi, A., Aqlan, M.H.: A coupled system of Caputo-type sequential fractional differential equations with coupled (periodic/anti-periodic type) boundary conditions. Mediterr. J. Math. 14, Article ID 227 (2017)

25. Fečkan, M., Marynets, K.: Approximation approach to periodic BVP for mixed fractional differential systems. J. Comput. Appl. Math. 339, 208-217 (2018)

26. Bai, C.Z.: Impulsive periodic boundary value problems for fractional differential equation involving Riemann-Liouville sequential fractional derivative. J. Math. Anal. Appl. 384, 211-231 (2011)

27. Belmekki, M., Nieto, J.J., Rodríguez-López, R.: Existence of solution to a periodic boundary value problem for a nonlinear impulsive fractional differential equation. Electron. J. Qual. Theory Differ. Equ. 2014, 16 (2014)

28. Wei, Z.L., Dong, W., Che, J.L.: Periodic boundary value problems for fractional differential equations involving a Riemann-Liouville fractional derivative. Nonlinear Anal. 73, 3232-3238 (2010)

29. Benchohra, M., Bouriah, S., Graef, J.R.: Nonlinear implicit differential equations of fractional order at resonance. Electron. J. Differ. Equ. 2016, 324 (2016)

30. Benchohra, M., Bouriah, S., Nieto, J.J.: Existence of periodic solutions for nonlinear implicit Hadamard's fractional differential equations. Rev. R. Acad. Cienc. Exactas Fís. Nat., Ser. A Mat. 112, 25-35 (2018)

31. Benchohra, M., Bouriah, S., Graef, J.R.: Boundary value problems for nonlinear implicit Caputo-Hadamard-type fractional differential equations with impulses. Mediterr. J. Math. 2017, 14 (2017)

32. Nieto, J., Ouahab, A., Venktesh, V.: Implicit fractional differential equations via the Liouville-Caputo derivative. Mathematics 3, 398-411 (2015)

33. Ali, Z., Zada, A., Shah, K.: On Ulam's stability for a coupled systems of nonlinear implicit fractional differential equations. Bull. Malays. Math. Sci. Soc. (2018). https://doi.org/10.1007/s40840-018-0625-x

34. Kucche, K.D., Nieto, J.J., Venktesh, V.: Theory of nonlinear implicit fractional differential equations. Differ. Equ. Dyn. Syst. (2016). https://doi.org/10.1007/s12591-016-0297-7

35. Vivek, D., Kanagarajan, K., Elsayed, E.M.: Nonlocal initial value problems for implicit differential equations with Hilfer-Hadamard fractional derivative. Nonlinear Anal. 23, 341-360 (2018)

36. Vivek, D., Kanagarajan, K., Elsayed, E.M.: Some existence and stability results for Hilfer-fractional implicit differential equations with nonlocal conditions. Mediterr. J. Math. 2018, 15 (2018)

37. Nirmala, R.J., Balachandran, K.: The controllability of nonlinear implicit fractional delay dynamical systems. Int. J. Appl. Math. Comput. Sci. 27, 501-513 (2017)

38. Ahmad, N., Ali, Z., Shah, K., Zada, A., Rahman, G.U.: Analysis of implicit type nonlinear dynamical problem of impulsive fractional differential equations. Complexity 2018, Article ID 6423974 (2018) 
39. Abbas, S., Benchohra, M., Graef, J.R., Henderson, J.: Implicit Fractional Differential and Integral Equations: Existence and Stability. De Gruyter, Berlin (2018)

40. Hoang, D.H., Nhan, L.C., Truong, L.X.: Solvability of fractional differential equation with nonlocal boundary conditions at resonance. Vietnam J. Math. 45, 625-638 (2017)

41. Chen, Y., Tang, X.H.: Solvability of sequential fractional order multi-point boundary value problems at resonance. Appl. Math. Comput. 218, 7638-7648 (2012)

42. Ge, F.D., Zhou, H.C.: Existence of solutions for fractional differential equations with three-point boundary conditions at resonance in $\mathbb{R}^{n}$. Electron. J. Qual. Theory Differ. Equ. 2014, 68 (2014)

43. Zhang, Y.H., Bai, Z.B., Feng, T.T.: Existence results for a coupled system of nonlinear fractional three-point boundary value problems at resonance. Comput. Math. Appl. 61, 1032-1047 (2011)

44. Bai, Z.B., Lü, H.S.: Positive solutions for boundary value problem of nonlinear fractional differential equation. J. Math Anal. Appl. 311, 495-505 (2005)

45. O'Regan, D., Chao, Y.J., Chen, Y.Q.: Topological Degree Theory and Application. Taylor \& Francis, Boca Raton (2006)

46. Mawhin, J.: Topological Degree Methods in Nonlinear Boundary Value Problems, Expository Lectures from the CBMS Regional Conference Held at Harvey Mudd. CBMS Regional Conference Series in Mathematics. Am. Math. Soc., Providence (1979)

47. Mawhin, J.: Topological degree and boundary value problems for nonlinear differential equations. In: Topological Methods for Ordinary Differential Equations, Montecatini Terme, 1991. Lecture Notes in Math., vol. 1537, pp. 74-142. Springer, Berlin (1993)

\section{Submit your manuscript to a SpringerOpen ${ }^{\circ}$ journal and benefit from:}

- Convenient online submission

- Rigorous peer review

- Open access: articles freely available online

- High visibility within the field

- Retaining the copyright to your article

Submit your next manuscript at $\boldsymbol{~ s p r i n g e r o p e n . c o m ~}$ 